\title{
Incorporating interface affected zones into crystal plasticity
}

\author{
J. R. Mayeur*, I. J. Beyerlein, C. A. Bronkhorst, and H. M. Mourad \\ Theoretical Division, Los Alamos National Laboratory, Los Alamos, NM 87545
}

\begin{abstract}
This work presents a crystal plasticity modeling framework that accounts for the influence of material interfaces on the plastic behavior of the two crystals on either side of the interface. Within an interfaceaffected zone (IAZ) extending from both sides of the interface, slip system activity is presumed to be biased towards systems that permit slip transfer across the interface. The preferred slip transfer pathways are determined from the geometric alignment of the slip systems and the stress state within each crystal. The IAZ model is applied to study the plastic stability of $\mathrm{Cu}-\mathrm{Nb}$ bicrystals under plane strain compression. Our results show that the additional constraints imposed through the enforcement of slip continuity across the interface leads to reduced plastic stability as compared to the case without an IAZ for several of the interfaces studied.
\end{abstract}

Keywords: crystal plasticity, nanolamellar composites, interface stability, slip transfer, accumulative roll bonding

\section{Introduction}

The importance of material interfaces on the plastic deformation of polycrystalline (single and/or multiphase) metals has long been established (Chalmers, 1949; Hirth, 1972; Mishin et al., 2010). The interface is a region between two adjoining crystals that are dissimilar in orientation (grain boundary) and/or structure (e.g., fcc/fcc or fcc/bcc interface). In the presence of an applied stress or strain field, interfaces are capable of acting as dislocation sources (Beyerlein et al., 2011a, 2013c; Hunter and Beyerlein, 2013; Keh and Weissmann, 1963; Spearot et al., 2007; Li, 1963), sinks (Armstrong et al., 1962; Hirth, 1972; Li, 1963; Misra, 2006), and obstacles to slip. Under certain conditions some materials can emit twins (Beyerlein et al., 2011a; Niezgoda et al., 2014; Wang et al., 2010a,b; Zhu et al., 2012; Adams et al., 2012) from interfaces. These behaviors depend on interface crystallography, morphology, defect content, and chemistry. Thus, the properties of interfaces play an important role in governing deformation mechanisms at a small scale as well as the aggregate response at the polycrystal scale.

Over the past several years, there have been many attempts to engineer the properties of interfaces to optimize polycrystal performance. This approach is most often applied to nanocrystalline metals, which contain a high density of interfaces (Beyerlein et al., 2013b; Chookajorn et al., 2012; Gleiter, 2008; Lu et al., 2009; Zheng et al., 2012). Most of these efforts have been experimentally driven or involved computational modeling at the atomic scale. Mechanics-based crystal plasticity modeling at the mesoscale is another important tool that can be used to design and study interface effects and has the potential to connect interface-driven deformation mechanisms to aggregate polycrystalline response. However, most crystal plasticity modeling frameworks treat material interfaces in a simplified manner neglecting many of the relevant physical processes occurring at this scale. With recent advances in computational power and crystal plasticity modeling techniques that account for the realistic topology and microstructure of polycrystals (Ardeljan et al., 2014; Hansen et al., 2013; Lebensohn, 2001; Roters et al., 2010), these simplified treatments are no longer a practical necessity.

At present, in spatially resolved polycrystal models (i.e., those wherein each grain and/or phase is explicitly represented) that use classical single crystal plasticity, the interface kinematics are such that the crystals are considered to be either bonded or allowed to undergo interfacial sliding. The former is

\footnotetext{
* corresponding author

Email address: jmayeur@lanl.gov (J. R. Mayeur)
} 
represented through the enforcement of continuity of tractions and displacements at the interface. While these are undoubtedly important constraints, any additional constraints associated with interfaces are still largely neglected. Slip system activity is commonly governed by a flow rule that is not directly influenced by the interface other than through the evolution of the local stress and internal state variables. In essence, the polycrystal is represented by an aggregate of crystals that do not communicate with each other except through prescribed equilibrium and displacement boundary conditions at the interfaces.

In the present work, we develop a model in which the slip activity in regions adjacent to a material interface is affected by the state of the material on the other side of the interface. Within a region extending into the crystals on both sides of the interface, referred to as an interface-affected zone (IAZ), slip is biased toward slip systems that are well coordinated across the interface. The IAZ model is incorporated into a spatially resolved crystal plasticity finite element framework to model a bicrystal under plane strain compression. These calculations are designed to reveal the effects of the IAZ on slip activity, lattice reorientation, and crystallographic stability of interface character with respect to deformation.

In the following, we begin with a physical description of slip transfer across an interface and a review of prior model developments that incorporate this mechanism for grain boundaries. Next, we present our model for slip transfer within an IAZ and its application for understanding the crystallographic stability of a bimetal interface in large strain deformation. Last, we present simulation results and their implications with respect to recent experimental observations. Although our interests are focused on bimetal interfaces, the IAZ modeling framework presented here can apply to homophase (grain boundaries) and heterophase interfaces alike.

\subsection{Slip continuity across an interface}

An important interface-related deformation mechanism involves a combination of interface-driven unit processes, variously called slip transfer; slip, slip band, or glide continuity across an interface; slip transmission; or dislocation interface crossing (Clark et al., 1992; Davis et al., 1966; Embury and Hirth, 1994; Lee et al., 1990a; Louat, 1985; Robertson et al., 1989; Werner and Prantl, 1990). Slip transfer or slip continuity, as we elect to call it here, is a mechanism for facilitating plastic deformation in polycrystalline metals. Some interfaces are more efficient than others at transferring slip and in this way, interfaces can profoundly affect plastic flow and ductility. Given its importance, many experimental studies on polycrystalline metals have studied slip continuity across grain boundaries (Hook and Hirth, 1967a,b; Patriarca et al., 2013; Šittner and Paidar, 1989; Šittner et al., 1992; Wang et al., 2010a,b; Werner and Prantl, 1990).

Continuing or transferring slip does not necessarily involve the exchange, flow, or flux of dislocations from one crystal to another. Most generally it proceeds by a sequence of events, in which slip of one crystal into the interface promotes slip in the adjoining crystal from the interface. As such, continuing slip across an interface can be envisioned to take place in several ways. For instance, dislocations belonging to one slip system $\alpha$ in crystal $A$ can pile up at an interface and invoke nucleation of dislocations on another slip system $\beta$ on the other side in crystal $B$. Alternatively, the stress field of approaching $\alpha$ dislocations in crystal $A$ can promote nucleation of $\beta$ dislocations on the other side in crystal $B$. Also impinging $\alpha$ dislocations in crystal $A$ can be absorbed into the interface and induce emission of $\beta$ dislocations into crystal $B$ in order to lower interface energy. The above scenarios have been used in prior studies to describe slip transmission (Beyerlein et al., 2013b; Louat, 1985; Ma et al., 2006; Patriarca et al., 2013; Roters et al., 2010; Seal et al., 2012; Wang et al., 2008, 2010a,b).

Slip continuity is not equally probable among all pairs of $\alpha-\beta$ slip systems across an $A-B$ interface. Its propensity is believed to be strongly biased towards certain pathways. That is, for some pairs of slip systems the interface permits slip continuity, while the same interface is a strong obstacle for others. Several experimental and analytical studies have been dedicated to identifying these dependencies, mostly for grain boundaries (Lee et al., 1990a,b; Clark et al., 1992; Bieler et al., 2009; Werner and Prantl, 1990), but also some studies have examined bimetal interfaces (Beyerlein et al., 2013b; Louat, 1985; Ma et al., 2006; Patriarca et al., 2013; Roters et al., 2010; Seal et al., 2012; Wang et al., 2008, 2010a,b). Most of these works adapt and/or build upon the criteria of Werner and Prantl (1990) and Lee-Roberston-Birnbaum (LRB) Lee et al. (1990a,b); Clark et al. (1992).

In light of these works, the consensus is that efficient slip pathways for continuing slip are well coordinated spatially, meeting within the same general vicinity at the interface; geometrically, having 
well aligned slip planes and slip vectors; and mechanically, with driving forces directed into and out of the interface. Consequently, the number of such favorable pathways depends on the crystallographic character (5-parameter description including the orientation relationship and interface plane) of the interface and its orientation relationship with the loading state. An interface character that provides a significant barrier to slip has little to no efficient transmission pathways across it, or vice versa. An interface character that readily transfers slip has many favorable transmission pathways and provides little resistance to slip. Note that interface character will generally change during deformation due to the lattice reorientation of the adjoining crystals. Thus, it is possible that an interface can evolve with deformation to be more or less favorable for slip transfer.

On this basis, we can anticipate some consequences of the bias posed by slip continuity at an interface. First, the interface character can clearly affect the slip activity of its adjoining crystals. Second, by virtue of a change in slip activity, the deformation response and in particular, the orientational stability of crystals joined to an interface will not be the same as their respective single crystal orientations. Third, interface-affected slip activity is expected to extend a finite amount from the interface. Thus, within an IAZ, slip is predominantly controlled by the interface but the influence diminishes as a function of distance from the interface. Last, slip within the IAZ is more constrained compared to that of the bulk and hence it is expected that some subset of interfaces that are stable when there is no IAZ will become unstable in the presence of an IAZ. A stable interface is one in which the interface character does not change with continued straining.

\subsection{Prior work on slip transmission}

A few groups have studied slip continuity across grain boundaries using crystal plasticity modeling (Ashmawi and Zikry, 2002; Cermelli and Gurtin, 2002; Ma et al., 2006; Acharya, 2007; Lim et al., 2011; Ekh et al., 2011; Voyiadjis et al., 2014). Ma et al. (2006) developed a crystal plasticity framework with a special interface finite element that has a different flow resistance than the grain interior elements, which is a core-mantle type of approach. The interface elements have an elevated critical resolved shear stress that is a function of the residual Burgers vector that is left behind in the interface after a successful transmission event. In this way, slip pathways with a high degree of misalignment are penalized and those that are well-aligned are favored. An alternative approach put forth by Ashmawi and Zikry (2002) captured slip continuity across grain boundaries by introducing a coupled set of evolution equations for the mobile and immobile dislocation density populations into a standard crystal plasticity framework. The evolution of the mobile dislocation density throughout the material is tracked on an element-byelement basis. With regard to the mobile dislocation densities interaction with interfaces, three different scenarios are considered: 1) full transmission, 2) partial transmission and partial blockage, and 3) fully blocked. The dislocation-interface interactions are in essence determined by the LRB criteria (Lee et al., 1990a,b; Clark et al., 1992). Cermelli and Gurtin (2002) and Evers et al. (2004) addressed the issue by developing nonlocal extensions of classical single crystal plasticity to account for size-dependent hardening (e.g., GNDs). Both frameworks are higher-order gradient theories that allow for the direct application of boundary conditions on plastic degrees-of-freedom (e.g. plastic strains and/or their conjugate fluxes) at the grain boundary. The primary distinction between the two modeling frameworks is that the former (Cermelli and Gurtin, 2002) contains higher-order stresses (work-conjugate higher-order theory), whereas the model of Evers et al. (2004) does not (non-work-conjugate).

Compared to grain boundaries, bimetal (heterophase) interfaces have proven to be much stronger obstacles to slip transfer. Slip transfer is thought to prevail across grain boundaries regardless of the grain size. However, it is uncertain whether slip transfer is operative across bimetal interfaces (Werner and Stüwe, 1985a,b) and if so, it is believed to occur at a much higher critical stress than what is required for transfer through grain boundaries. Multilayered bimetal films of various material systems have been tested for size-dependent hardness (Misra, 2006; Monclús et al., 2013; Schweitz et al., 2001) and in a few cases size-dependent strength (Beyerlein et al., 2013a; Huang and Spaepen, 2000; Mara et al., 2008). Using dislocation-based modeling, it was concluded that slip transfer is predominant only at the very finest nanolayer thicknesses ( $2-5 \mathrm{~nm}$ ) where sufficiently high stresses are achieved (Misra et al., 2005). The films studied possessed interfaces of predominantly one character (Lee et al., 2012). Multilayered bimetal sheets have recently been fabricated by bulk-scale metal working techniques, such as rolling and accumulative roll bonding (Beyerlein et al., 2013a; Carpenter et al., 2014b), from which bimetal interfaces of different crystallographic character emerge (Beyerlein et al., 2014b). It is possible that slip 
transfer could occur at higher length scales for these different interface characters in combination with the high stress states experienced during severe plastic deformation. Currently, the operative deformation mechanisms in submicron and nanocrystals joined to bimetal interfaces have yet to be clarified and are not directly accessible via experiment.

\subsection{Objectives of the current work}

Herein, we propose a crystal plasticity modeling framework that modifies the slip activity in crystals attached to an interface according to the availability of favorable slip transfer pathways. The effects of the interface are assumed to occur within an IAZ extending from both sides of the interface. Our IAZ model shares some similarities to the approaches of Ashmawi and Zikry (2002) and Ma et al. (2006) in that we use modified constitutive properties in regions near interfaces based on the crystallographic misalignment of neighboring crystals. Specifically, within the IAZ, the slip resistance on systems that do not comprise an efficient transfer pathway is elevated in an effort to bias slip towards favorable pathways. The baseline constitutive model adopts a homogeneous viewpoint of slip, where the motion of dislocation distributions is treated. The dislocations involved in slip outside the IAZ and in the transfer of slip across an interface inside the IAZ are, therefore, not modeled discretely. Accordingly, we will not be concerned with how slip transfer occurs at the scale of an individual dislocation. In other words, the outcomes of particular dislocation reactions, such as those between an interface misfit dislocation and a run-in dislocation, the screw/edge character of the reacting dislocations, or the effects of residuals remaining in the interface, will not be handled explicitly. Here we will be concerned with the geometric and mechanical conditions that favor slip transfer at a mesoscopic scale.

The proposed modeling framework is applicable to both grain boundaries and heterophase interfaces, however, we are motivated by an interest in studying the crystallographic stability of bimetal interfaces in large strain deformation. Toward this end, our investigations will examine how slip activity and grain reorientation of the crystals adjoined to an interface are affected by considering the IAZ. Simulation results are compared to the prior case in which slip at a bonded interface was not coordinated across the interface and hence less constrained. The results provide insight into the dominant deformation processes occurring in these materials and the stability of specific interface characters.

\section{Model formulation}

\subsection{Kinematics}

In the following, we outline the basic kinematic relations that apply to all material points. The standard kinematic assumptions of classical single crystal plasticity based on a two-term multiplicative decomposition of the deformation gradient into elastic, $\mathbf{F}^{e}$, and plastic, $\mathbf{F}^{p}$, parts, i.e.,

$$
\mathbf{F}=\mathbf{F}^{e} \mathbf{F}^{p}
$$

Dislocation motion, which leaves lattice vectors unaltered with respect to their reference state, is encapsulated by $\mathbf{F}^{p}$ and the lattice stretch and rotation (including superposed rigid rotations) is given by $\mathbf{F}^{e}$. $\mathbf{F}^{p}$ evolves with dislocation motion within their glide planes and is defined by the plastic velocity gradient with respect to the fixed isoclinic intermediate configuration as

$$
\overline{\mathbf{L}}^{p}=\dot{\mathbf{F}}^{p} \mathbf{F}^{p^{-1}}=\sum_{\alpha} \dot{\gamma}^{\alpha} \overline{\mathbf{s}}^{\alpha} \otimes \overline{\mathbf{n}}^{\alpha}
$$

where $\alpha$ denotes the slip system, $\dot{\gamma}^{\alpha}$ is the slip system shear rate, $\overline{\mathbf{s}}^{\alpha}$ is the unit slip vector in the intermediate configuration, and $\overline{\mathbf{n}}^{\alpha}$ is the unit slip plane normal. The unit slip and normal vectors for a given slip system are mapped to the current configuration as

$$
\mathbf{s}^{\alpha}=\mathbf{F}^{e} \overline{\mathbf{s}}^{\alpha} \quad, \quad \mathbf{n}^{\alpha}=\mathbf{F}^{e^{-T}} \overline{\mathbf{n}}^{\alpha}
$$

Equation 3 enables one to track the crystallographic orientation evolution, which is embedded in the rotational part of $\mathbf{F}^{e}$, i.e. $\mathbf{R}^{e}=\mathbf{F}^{e} \mathbf{U}^{e^{-1}}$ where $\mathbf{U}^{e}$ is the elastic right Cauchy stretch tensor. 


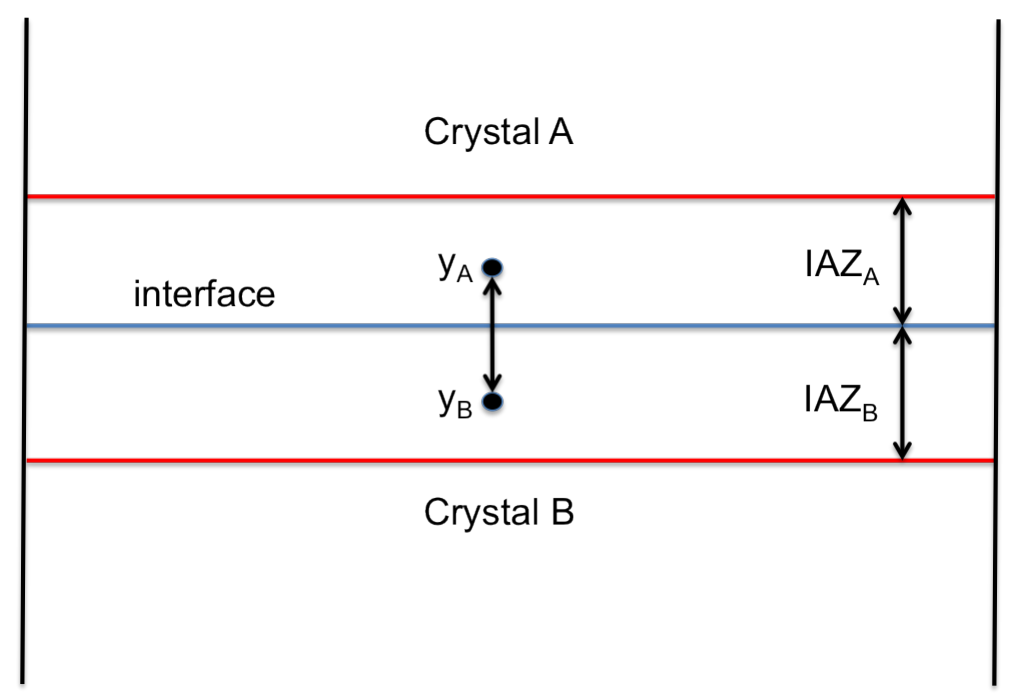

Figure 1: Schematic illustrating the interface affected zone (IAZ).

\subsection{Slip kinetics and the $I A Z$}

Central to the model is the concept of an IAZ, which extends into the crystallite interiors adjacent to the interface as shown in Fig. 1. Material points within the IAZ $(y \in$ IAZ) have different constitutive behavior than those outside of this region $(x \notin \mathrm{IAZ})$. The difference is encapsulated entirely within the constitutive prescription of $\dot{\gamma}^{\alpha}$. For material points outside of the IAZ denoted by $x$, the expression for the slip system shear rate is a function of only local spatial information at that material point, which we express symbolically as $\dot{\gamma}^{\alpha}=f^{\alpha}(x)$.

The slip rate on each slip system is determined by the thermodynamics of dislocation glide (Kocks and Mecking, 2003). Several models have been proposed that link $\dot{\gamma}^{\alpha}$ to thermally activated dislocation motion (Beyerlein et al., 2011b; Meissonnier et al., 2001). In the present model we adopt the kinetics law detailed in Bronkhorst et al. (2007).

$$
\dot{\gamma}^{\alpha}(x)=\dot{\gamma}_{0} \exp \left[-\frac{F_{0}}{k \theta(x)}\left\langle 1-\left\langle\frac{\left|\tau^{\alpha}(x)\right|-s^{\alpha}(x) \mu^{*}(x)}{g^{\alpha} \mu^{*}(x)}\right\rangle^{p}\right\rangle^{q}\right] \operatorname{sgn}\left(\tau^{\alpha}(x)\right)
$$

where $\dot{\gamma}_{0}$ is the reference shearing rate, $F_{0}$ is the activation free energy, $k$ is Boltzmann's constant, $\theta$ is the absolute temperature, $\mu^{*}=\mu / \mu_{0}$ is the normalized shear modulus, $\tau^{\alpha}$ is the resolved shear stress, $s^{\alpha}$ is the athermal slip resistance due to dislocation storage, $g^{\alpha}$ is the drag stress, and $p$ and $q$ are exponents that characterize the shape of the obstacle profile. The normalized shear modulus is defined in terms of $\mu$, the temperature-dependent shear modulus, and the shear modulus at $0 \mathrm{~K}, \mu_{0}$. The athermal slip resistance evolves according to the following equations

$$
\begin{gathered}
\dot{s}^{\alpha}(x)=\sum_{\beta} h^{\alpha \beta}(x)\left|\dot{\gamma}^{\beta}(x)\right| \quad, \quad s^{\alpha}(t=0)=s_{0} \\
h^{\alpha \beta}(x)=h_{0}\left[r+(1-r) \delta_{\alpha \beta}\right]\left(\frac{\hat{s}^{\beta}(x)-s^{\beta}(x)}{\hat{s}^{\beta}(x)-s_{0}^{\beta}}\right) \\
\hat{s}^{\beta}(x)=\hat{s}_{0}\left(\frac{\left|\dot{\gamma}^{\beta}(x)\right|}{\dot{\gamma}_{0}}\right)^{\frac{k \theta(x)}{A}}
\end{gathered}
$$

where $s_{0}$ is the initial slip resistance, $h^{\alpha \beta}$ is the hardening interaction matrix, $r$ is the latent hardening ratio, $h_{0}$ is the initial hardening modulus, $\hat{s}^{\beta}$ is the saturated slip resistance, and $\hat{s}_{0}$ and $A$ are material parameters that control the rate and temperature dependence of the saturated slip resistance. Eqs. (4-7),

In contrast, for material points inside the IAZ denoted by $y$, the expression for the slip system shear rate is a function of local spatial information at that material point and averaged information from 
surrounding materials points on both sides of the interface (see Fig. 1). Consider the slip system shear rate at a material point $y_{A}$ inside the IAZ in crystal $A$, which can be expressed as

$$
\dot{\gamma}^{\alpha}\left(y_{A}, \bar{y}_{A}, \bar{y}_{B}\right)=\dot{\gamma}_{0} \exp \left[-\frac{F_{0}}{k \theta\left(y_{A}\right)}\left\langle 1-\left\langle\frac{\left|\tau^{\alpha}\left(y_{A}\right)\right|-s^{\alpha}\left(y_{A}\right) \mu^{*}\left(y_{A}\right)}{\hat{g}^{\alpha}\left(\bar{y}_{A}, \bar{y}_{B}\right) \mu^{*}}\right\rangle^{p}\right\rangle^{q}\right] \operatorname{sgn}\left(\tau^{\alpha}\left(y_{A}\right)\right)
$$

where $y_{B}$ is a material point in the IAZ in crystal $B$ that interacts with $y_{A}, \bar{y}_{A}$ and $\bar{y}_{B}$ are sets of points surrounding $y_{A}$ and $y_{B}$, respectively, over which information may be chosen to be averaged, and $\hat{g}^{\alpha}$ is a slip resistance modified by the interface. When $\hat{g}^{\alpha}$ is large, the activation energy for slip is higher, which implies that slip is less likely.

\subsection{Slip resistance within the $I A Z$}

The slip activity of material points within the IAZ is biased towards pairs of systems that form a favorable pathway for continuous slip across the interface. It is possible to envision a number of plausible and appropriate ways to enforce constraints on slip system activity within the IAZs. In this work, we elect to penalize unfavorable $A \rightarrow B$ pathways by increasing their slip resistances in comparison to favorable ones. The manner in which we effect this penalty via $\hat{g}^{\alpha}$ is discussed below. In the subsequent section, we present the criteria that are used to define a favorable pathway.

Consider two interacting points $y_{A}$ and $y_{B}$ belonging to the IAZ on opposite sides of the interface as shown in Fig. 1. These may be, for instance, two adjacent points that lie immediately on opposite sides of the interface and are presumably highly interacting. The intent is to substantially bias slip activity towards slip systems at material points $y_{A}$ and $y_{B}$ whose glide is continuous across the interface. To this end, we define a factor $P^{\alpha \beta}$ that measures the likelihood of slip continuity of an $\alpha \rightarrow \beta$ pathway, where $\alpha$ is the index of a slip system in crystal $A\left(\alpha \in N_{A}\right)$ and $\beta$ is the index of a slip system in crystal $B$ $\left(\beta \in N_{B}\right) . P^{\alpha \beta}$ ranges from 0 to 1 , with $P^{\alpha \beta}=1$ representing perfect continuity and $P^{\alpha \beta}=0$ a null chance of slip transfer along a given pathway. To reduce $\dot{\gamma}^{\alpha}$ on unfavorable systems, thereby forcing other slip systems to accommodate the imposed deformation, the drag stress $g^{\alpha}$ is elevated for pathways with low $P^{\alpha \beta}$ values, i.e., $P^{\alpha \beta}<P_{c r}$, where $P_{c r}$ is a threshold value. The expression for $\hat{g}^{\alpha}$ for a general slip system $\alpha \rightarrow \beta$ pathway through points $y_{A}$ and $y_{B}$ within the IAZ is given as

$$
\hat{g}^{\alpha}\left(\bar{y}_{A}, \bar{y}_{B}\right)=g^{\alpha} f\left(P^{\alpha \beta}\left(\bar{y}_{A}, \bar{y}_{B}\right)\right)
$$

where $0 \leq P^{\alpha \beta} \leq 1$ and $f\left(P^{\alpha \beta}=1\right)=1$. The analogous expression for slip transfer from crystal $B$ to crystal $A$ is expressed as $\hat{g}^{\beta}\left(\bar{y}_{B}, \bar{y}_{A}\right)$. The penalty function, $f$, is assumed to have the following simple form

$$
f\left(P^{\alpha \beta}\right)= \begin{cases}1 & \text { if } P^{\alpha \beta} \geq P_{c r} \\ M & \text { if } P^{\alpha \beta}<P_{c r}\end{cases}
$$

where $M$ is a constant penalty factor. Thus, $\hat{g}^{\alpha}$ is given as

$$
\hat{g}^{\alpha}\left(\bar{y}_{A}, \bar{y}_{B}\right)=\left\{\begin{array}{ll}
g^{\alpha} & \text { if } P^{\alpha \beta} \geq P_{c r} \\
M g^{\alpha} & \text { if } P^{\alpha \beta}<P_{c r}
\end{array} .\right.
$$

In applying the model in the following calculations, this particular choice of penalty function, $f$, (Eq. (10)) is combined with a sufficiently large value of $M$ in order to represent an extreme case where slip on systems that do not have good slip transfer characteristics is prohibited within the IAZ.

\subsection{Slip continuity factor $P^{\alpha \beta}$}

Two criteria are used to define $P^{\alpha \beta}$, reflecting whether a slip pathway across the interface is continuous. One is based on geometric compatibility and the other on the local stress state. In brief, the geometric component depends on the angle between the slip plane traces in the interface plane and the angle between the Burgers vectors. The stress-based component accounts for the magnitude and sign of the resolved shear stresses on the incoming $\alpha$ and outgoing $\beta$ slip planes. Consequently, $P^{\alpha \beta}$ is a dynamically evolving parameter that depends on the current orientation relationship and interface plane. Each component is presented in detail below. Designation of the functional dependence of $P^{\alpha \beta}$ on $\bar{y}_{A}$ and $\bar{y}_{B}$ is suppressed for the sake of brevity. 
First, slip continuity requires that the slip planes and slip vectors of the incoming $(\alpha)$ and outgoing $(\beta)$ slip systems are well aligned. For each potential slip transfer pathway from crystal $A$ to crystal $B$, the geometric compatibility factor, $\chi^{\alpha \beta}\left(\bar{y}_{A}, \bar{y}_{B}\right)$, is adopted from (Werner and Prantl, 1990), and given as

$$
\chi^{\alpha \beta}\left(\bar{y}_{A}, \bar{y}_{B}\right)=\cos \left[\frac{\pi}{2}\left(\frac{\theta^{\alpha \beta}\left(\bar{y}_{A}, \bar{y}_{B}\right)}{\theta_{C}}\right)\right] \cos \left[\frac{\pi}{2}\left(\frac{\kappa^{\alpha \beta}\left(\bar{y}_{A}, \bar{y}_{B}\right)}{\kappa_{C}}\right)\right]
$$

where $\theta^{\alpha \beta}\left(\bar{y}_{A}, \bar{y}_{B}\right)$ is the angle between slip plane traces of the incoming and outgoing systems in the interface plane and $\kappa^{\alpha \beta}\left(\bar{y}_{A}, \bar{y}_{B}\right)$ is the angle between incoming and outgoing slip vectors. The parameters $\theta_{c}$ and $\kappa_{c}$ define critical cut-off values for these angles above which transmission is not allowed, i.e., if $\kappa^{\alpha \beta}>\kappa_{c}$ or $\theta^{\alpha \beta}>\theta_{c} \Rightarrow \chi^{\alpha \beta}=0 . \chi^{\alpha \beta}\left(\bar{y}_{A}, \bar{y}_{B}\right)$ is a $N_{A} \times N_{B}$ matrix that represents the geometric favorability of all possible slip pathways from $y_{A}$ to $y_{B}$. The angle between slip plane traces, $\theta^{\alpha \beta}\left(\bar{y}_{A}, \bar{y}_{B}\right)$, in the interface plane is calculated as

$$
\begin{gathered}
\theta^{\alpha \beta}\left(\bar{y}_{A}, \bar{y}_{B}\right)=\arccos \left(\boldsymbol{\ell}^{\alpha}\left(\bar{y}_{A}\right) \cdot \boldsymbol{\ell}^{\beta}\left(\bar{y}_{B}\right)\right) \\
\boldsymbol{\ell}^{\alpha}\left(\bar{y}_{A}\right)=\frac{\mathbf{n}^{\alpha}\left(\bar{y}_{A}\right) \times \mathbf{n}_{i n t}}{\left|\mathbf{n}^{\alpha}\left(\bar{y}_{A}\right) \times \mathbf{n}_{\text {int }}\right|} \quad, \quad \boldsymbol{\ell}^{\beta}\left(\bar{y}_{B}\right)=\frac{\mathbf{n}^{\beta}\left(\bar{y}_{B}\right) \times \mathbf{n}_{i n t}}{\left|\mathbf{n}^{\beta}\left(\bar{y}_{B}\right) \times \mathbf{n}_{\text {int }}\right|}
\end{gathered}
$$

where $\ell^{\alpha}\left(\bar{y}_{A}\right)$ is the unit vector along the line of intersection of slip system $\alpha$ in crystal $A$ with the interface plane, $\ell^{\beta}\left(\bar{y}_{B}\right)$ is the unit vector along the line of intersection of slip system $\beta$ in crystal $B$ with the interface plane, and $\mathbf{n}_{\text {int }}$ is the unit vector normal to the interface pointing from crystal $A$ to crystal $B$. The angle between the Burgers vectors, $\kappa^{\alpha \beta}\left(\bar{y}_{A}, \bar{y}_{B}\right)$, is given by

$$
\kappa^{\alpha \beta}\left(\bar{y}_{A}, \bar{y}_{B}\right)=\arccos \left(\mathbf{s}^{\alpha}\left(\bar{y}_{A}\right) \cdot \mathbf{s}^{\beta}\left(\bar{y}_{B}\right)\right)
$$

where $\mathbf{s}^{\alpha}\left(\bar{y}_{A}\right)$ is the unit slip vector of slip system $\alpha$ in crystal $A$ and $\mathbf{s}^{\beta}\left(\bar{y}_{B}\right)$ is the unit slip vector of slip system $\beta$ in crystal $B$. Thus, $\chi^{\alpha \beta}$ assumes values between 0 and 1 .

Second, slip continuity is conditional on sufficient resolved shear stress to drive incoming and outgoing slip. Therefore, a second criterion is represented by the following set of constraints

$$
\begin{aligned}
& \left|\tau^{\alpha}\left(\bar{y}_{A}\right)\right| \geq \tau_{c r}\left(\bar{y}_{A}\right) \text { and } \mathbf{s}^{\alpha}\left(\bar{y}_{A}\right) \cdot \mathbf{n}_{i n t} \operatorname{sgn}\left(\tau^{\alpha}\left(\bar{y}_{A}\right)\right)>0 \\
& \left|\tau^{\beta}\left(\bar{y}_{B}\right)\right| \geq \tau_{c r}\left(\bar{y}_{B}\right) \text { and } \mathbf{s}^{\beta}\left(\bar{y}_{B}\right) \cdot \mathbf{n}_{i n t} \operatorname{sgn}\left(\tau^{\beta}\left(\bar{y}_{B}\right)\right)>0
\end{aligned}
$$

where the above expressions apply for slip transfer from crystal $A$ to crystal $B . \tau^{\alpha}\left(\bar{y}_{A}\right)$ is the resolved shear stress on slip system $\alpha$ in crystal $A, \tau^{\beta}\left(\bar{y}_{B}\right)$ is the resolved shear stress on slip system $\beta$ in crystal $B, \tau_{c r}\left(\bar{y}_{A}\right)$ is the critical slip transfer stress on slip system $\alpha$ in crystal $A$, and $\tau_{c r}\left(\bar{y}_{B}\right)$ is the critical slip transfer stress on slip system $\beta$ in crystal $B$. Here for simplicity, we set $\tau_{c r}$ equal to $s^{\alpha}$. Thus, $P^{\alpha \beta}$ is expressed as

$$
P^{\alpha \beta}= \begin{cases}\chi^{\alpha \beta} & \text { if }(16) \text { and }(17) \text { are true } \\ 0 & \text { otherwise }\end{cases}
$$

\section{Application to $\mathrm{Cu}-\mathrm{Nb}$ interfaces}

\subsection{Plastic stability of $\mathrm{Cu}-\mathrm{Nb}$ interfaces}

To demonstrate the model, we explore the deformation response of several $\mathrm{Cu}-\mathrm{Nb}$ bicrystals with different interface characters. This study is in part motivated by observations of the unusual texture evolution in $\mathrm{Cu}-\mathrm{Nb}$ submicron and nanolayered composites produced via accumulative roll bonding $(\mathrm{ARB})$. It has been observed that once the individual layers contained one grain through the thickness, many of the components expected in rolling of monophase $\mathrm{Cu}$ and $\mathrm{Nb}$ diminished in intensity, which resulted in unusually sharp, nearly single-crystal like textures (Lee et al., 2012; Carpenter et al., 2012b). This was unexpected since typical rolling textures for severely deformed $\mathrm{Cu}$, for instance, contain several rolling components (Hammelrath et al., 1991; Hansen et al., 2013). One distinct single crystal-like texture emerged when the layer thickness, $h$, was refined below $1 \mu \mathrm{m}$, and a separate distinct texture transition occurred when $h<50 \mathrm{~nm}$, which coincided with the onset of twinning in the $\mathrm{Cu}$ phase (Beyerlein et al., 2014a). Microstructural evaluation found that the former submicron-layered texture corresponds 
to a predominance of interfaces composed of $\mathrm{Cu}$ orientations lying between the $\mathrm{C}\{112\}\langle 111\rangle$ and $\mathrm{D}$ $\{4411\}\langle 11118\rangle$ orientations with the $\mathrm{Nb}$ orientation located at the I component $\{112\}\langle 110\rangle$, while the nanolayered texture corresponds to interfaces comprised of $\mathrm{Cu}$ orientations distributed between the $\mathrm{Z}$ and Goss orientations and the Nb orientation persistent at I. The texture measurements provide indirect evidence that the interface is influencing slip activity, however, the mechanism of influence is unknown and cannot be assessed directly via experiment.

The ARB interfaces mentioned above are summarized in Table 1. The character is represented by the crystallographic orientation of the two crystals the interface joins. Here, the crystal orientations are provided in the $\{\mathrm{ND}\}\langle\mathrm{RD}\rangle$ convention. The "dashed" shorthand notation used lists the orientation (or component) for the $\mathrm{Cu}$ crystal before the dash and then the orientation for the $\mathrm{Nb}$ crystal after the dash. For example, the $\mathrm{C}$-I interface refers to the $\mathrm{C}$ orientation of the $\mathrm{Cu}$ crystal and the I orientation of the $\mathrm{Nb}$ crystal. In a previous study, the single crystal constitutive law and modeling framework we employ here were used to examine interface crystallographic stability only under the kinematic constraint of perfect bonding with no IAZ (Beyerlein et al., 2014b,a; Mayeur et al., 2013, 2014). The prior calculations predicted that the five interfaces listed in Table 1 were either metastable (within ten degrees of their stable point) or perfectly stable.

Herein, we examine the plastic stability of these five interfaces in plane strain compression under the influence of an IAZ. We consider an infinitely layered material wherein only one grain spans each layer, which represents the fine layered composites. The unit cell is a bicrystal containing a single $\mathrm{Cu}-\mathrm{Nb}$ interface with a prescribed initial character (orientation relationship and interface plane). Because we have no a priori knowledge of the size of the IAZ, we assume that the IAZ spans the entire domain of both crystals.

Table 1: Interface Nomenclature and Orientation Relationships (ND) [RD].

\begin{tabular}{lccc}
\hline \hline Interface & $\mathrm{Cu}$ & $\mathrm{Nb}$ & classification \\
\hline C-I & $(\overline{1} 21)[11 \overline{1}]$ & $(1 \overline{2} \overline{1})[101]$ & metastable \\
D-I & $(\overline{4} \overline{4} 11)[11118]$ & $(1 \overline{2} \overline{1})[101]$ & metastable \\
Goss-I & $(101)[010]$ & $(1 \overline{2} \overline{1})[101]$ & stable \\
Z-I & $(51 \overline{5})[1 \overline{1} \overline{1}]$ & $(1 \overline{2} \overline{1})[101]$ & metastable \\
Brass-I & $(101)[\overline{1} 21]$ & $(1 \overline{2} \overline{1})[101]$ & stable \\
\hline \hline
\end{tabular}

\subsection{Model setup}

The periodic bicrystal configuration introduced by Mayeur et al. (2013) and shown in Fig. 2 is used. The model consists of a $\mathrm{Nb}$ crystal joined to a $\mathrm{Cu}$ crystal with the interface normal in the $x_{2}$-direction (ND), subjected to plane strain compression boundary conditions. Periodicity of the displacements is enforced (van der Sluis et al., 2000) in both the $x_{1}$ and $x_{2}$ directions and the boundary conditions at the control vertices are illustrated in the schematic. Displacements are fully constrained at vertex 1 , the $x_{2}$ displacement component is constrained at vertex 2 , and the $x_{1}$ component of displacement is fixed at vertex 4. Loading is applied at vertex 4 via displacement control at a constant strain rate of $0.08 \mathrm{~s}^{-1}$.

The previously outlined crystal plasticity model is incorporated into an Abaqus/Standard Version 6.9.1 (2007) user material subroutine (UMAT). The baseline single crystal constitutive parameters, listed in Table 2, used for the constituent phases were determined by calibrating polycrystal simulations of each phase with no IAZ to experimental data (Hansen et al., 2013). The $\{111\}\langle 110\rangle$ slip systems for $\mathrm{Cu}$ and the $\{110\}\langle 111\rangle$ and $\{112\}\langle 111\rangle$ slip systems for $\mathrm{Nb}$ are considered. The slip system nomenclature is defined in Tables A.4 and A.5, which are given in the Appendix. The constitutive model is nonlocal in that the slip kinetics of a material point within the IAZ depend on material point information in neighboring finite elements. To simplify the constitutive update, the nonlocal variables (i.e. the crystallographic orientations and stress state) required for the calculation of $P^{\alpha \beta}$ are computed using values from the previously converged time step and held fixed for all subsequent plasticity iterations for the current time increment.

In the simulations with an IAZ, the IAZ spans the entire volume of crystal $A$ and crystal $B$, respectively. These choices were presumed to adequately represent submicron and nano-sized crystals, which do not develop substantial field gradients. In reality, the constraint imposed by an IAZ lies between two 


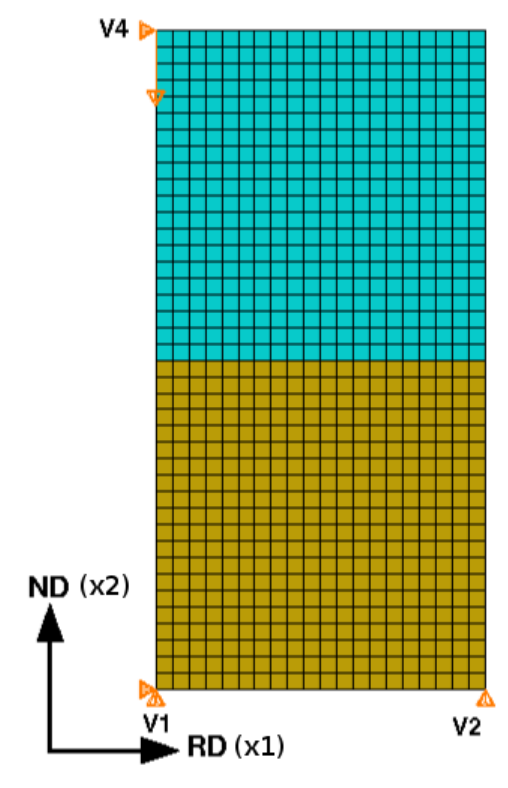

Figure 2: Periodic bicrystal finite element model.

extremes: (1) an IAZ with maximum constraint, in which slip systems that are not part of favorable slip transfer pathways are suppressed or (2) an IAZ with no constraint, which allows for all slip systems to potentially be active. In this first application of the IAZ model, we considered these two extreme cases to simplify the interpretation of the results. Intermediate conditions between these two extremes may be represented by modifying $M$ and $P_{c r}$. Recall, $M$ and $P_{c r}$ are, respectively, the penalty factor applied to the nominal drag stress for unfavorable slip systems and the critical value of the transmission factor for determining which pathways are penalized (Eq. (11)). For the maximum constraint case, we set to a large value $M=10^{3}$ and $P_{c r}$ was specified such that at least two slip systems could potentially be activated according to the initial geometric compatibility factor. The values of $P_{c r}$ used for each interface are given in Table 3.

\subsection{Assumptions}

For simplicity in demonstration, a number of assumptions are made with respect to the following calculations. Foremost is the assumption that the IAZ spans the entire grain. Additionally, we confine ourselves to the case in which only coordinated slip activity on either side of the interface within the IAZ is favored; other interface driven processes are not considered. For example, independent nucleation from extrinsic sources contained in the interface are not considered (Zhang et al., 2012). Any possible dependence of $\hat{g}$ on interface character is also omitted. Further, the processes involved in maintaining slip continuity across an interface are likely to be thermally activated (Monclús et al., 2013), implying that the penalty factor, $M$, can generally change with temperature and strain rate. However, since temperature and strain rate are fixed in our simulations, we do not assign a particular dependency of $M$ on these variables. Lastly, the interface formation energy, which is another important variable that contributes to determining the crystallorgraphic stability of an interface (Beyerlein et al., 2014b), is at present neglected for simplicity.

\subsection{Initial assessment based on prior calculations without an IAZ}

In previous calculations without an IAZ, we determined the active slip systems in $\mathrm{Cu}$ and $\mathrm{Nb}$ under large strain rolling. These interfaces were found to be either metastable (requiring slight reorientations to reach a stable end character) or stable. To gain initial insight prior to carrying out bicrystal simulations with an IAZ, we determine how many active slip system pairs are suitably connected across the interface $\left(\chi^{\alpha \beta}>0\right)$ in the calculations without an IAZ. Figure 3 charts the number of such slip systems pairs for the 
Table 2: Constitutive Model Parameters

\begin{tabular}{lccc}
\hline \hline Parameter & $\mathrm{Cu}$ & $\mathrm{Nb}$ & $\mathrm{units}$ \\
\hline$\rho$ & 8960 & 8400 & $\mathrm{~kg} \mathrm{~m}^{-3}$ \\
$c_{p}$ & 380 & 268 & $\mathrm{~J} \mathrm{~kg}^{-1} \mathrm{~K}^{-1}$ \\
$\alpha$ & 17.7 & 8.7 & $\mu \mathrm{m} \mathrm{m}^{-1} \mathrm{~K}^{-1}$ \\
$\eta$ & 0 & 0 & - \\
$m_{11}$ & -36.3 & -17.7 & $\mathrm{MPa} \mathrm{K}^{-1}$ \\
$C_{11_{0}}$ & 179.5 & 242.0 & $\mathrm{GPa}^{-1}$ \\
$m_{12}$ & -16.4 & 1.92 & $\mathrm{MPa} \mathrm{K}^{-1}$ \\
$C_{12_{0}}$ & 126.4 & 121.2 & $\mathrm{GPa}$ \\
$m_{44}$ & -25.7 & 2.18 & $\mathrm{MPa} \mathrm{K}^{-1}$ \\
$C_{44_{0}}$ & 82.5 & 28.0 & $\mathrm{GPa}$ \\
$r$ & 1.4 & 1.4 & - \\
$\dot{\gamma}_{0}$ & $10^{7}$ & $10^{7}$ & $\mathrm{~s}$ \\
$s_{0}$ & 1 & 40 & $\mathrm{MPa}$ \\
$g$ & 20 & 700 & $\mathrm{MPa}$ \\
$F_{0}$ & $1.0 \times 10^{-18}$ & $2.1 \times 10^{-19}$ & $\mathrm{~J}$ \\
$p$ & 0.33 & 0.34 & - \\
$q$ & 1.66 & 1.66 & - \\
$\hat{s}_{0}$ & 205 & 140 & $\mathrm{MPa}$ \\
$h_{0}$ & 200 & 300 & $\mathrm{MPa}$ \\
$A$ & $1.5 \times 10^{-19}$ & $1.0 \times 10^{-17}$ & $\mathrm{~J}$ \\
\hline \hline
\end{tabular}

Table 3: $P_{c r}$ values used in the IAZ simulations

\begin{tabular}{cccccc}
\hline \hline & C-I & D-I & Goss-I & Z-I & Brass-I \\
\hline$P_{c r}$ & 0.6 & 0.7 & 0.8 & 0.8 & 0.4 \\
\hline \hline
\end{tabular}

five interface characters in Table 1. In all cases, the enforcement of the slip continuity constraint reduces the number of active slip systems. As seen in the chart, only two or three of the active slip systems in the $\mathrm{Cu}$ crystal belong to continuous pathways for each interface, whereas little to no active slip systems in the $\mathrm{Nb}$ crystal for the C-I, D-I and Brass-I interfaces are favorably aligned. Only the Goss-I and Z-I interfaces have $\geq 2$ slip systems that could participate in continuous glide across the interface. While these interpretations are not based on calculations with an IAZ, they suggest that requiring both codeformation (perfectly bonded interface) and slip continuity across an interface would severely constrain the dynamics of deformation. Therefore, among these presumably five stable interfaces, only the Goss-I and Z-I interfaces are likely to be potentially stable when slip continuity is enforced. This also implies that the number of active slip systems would need to increase or change under the influence of the IAZ constraint from those found to be operative in simulations without an IAZ. With a potential change in slip activity, it is anticipated that the plastic stability of crystals affected by an IAZ can be significantly different from those not affected by one.

\section{Results with an IAZ}

In the following, we present and discuss the results of the simulations of the five interfaces considered both with and without an IAZ. First, we contrast the slip activities (below and in the Appendix) and the lattice reorientation via inverse pole figures. As a part of the discussion, we refer to various interfaces as either being stable, metastable, or unstable. A more quantitative discussion on the stability of these interfaces is provided in Section 5.

\subsection{Submicron interfaces: $C-I$ and $D-I$}

To examine the effect of the IAZ, we compare the crystal slip activities for the C-I interface with and without the IAZ. The C-I interface is selected since it is the most frequently occurring interface in the 


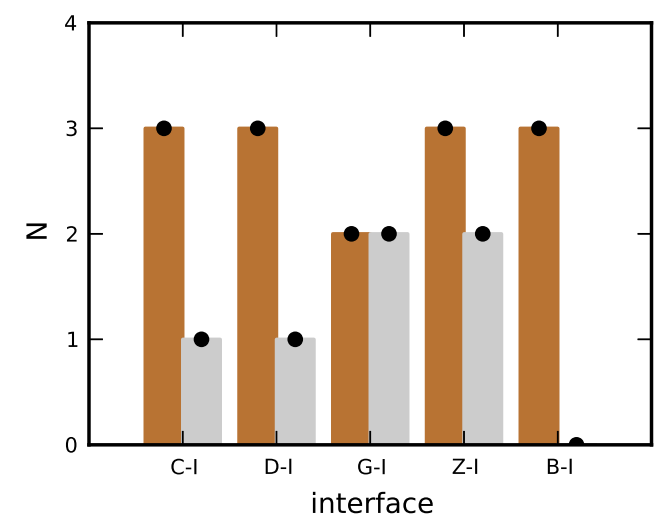

Figure 3: Number of slip systems in each crystal that have nonzero geometric transmission factors. The copper bar represents the copper crystal and the gray bar represents the $\mathrm{Nb}$ crystal

ARB material over a broad range of micron and submicron layer thicknesses (Beyerlein et al., 2014b; Lee et al., 2012). Fig. 4 compares the accumulated slip strains (plotted as averages over the entire crystal) on the active slip systems as a function of height reduction for simulations with and without an IAZ. The results clearly show that different slip system activities are predicted. For the simulations without an IAZ, two sets of slip systems are identically activated in the $\mathrm{Cu}$ crystal, which is indicative of symmetric slip. For the simulations with an IAZ, the slip symmetry is broken due to the activation of several more systems, albeit with relatively small amounts of accumulated slip. In the Nb crystal with no IAZ, three primary systems are activated, with each accommodating a substantial amount of the deformation. In contrast, a completely new set of slip systems is activated when there is an IAZ, and deformation is distributed among many more active slip systems of lesser amounts of accumulated slip as compared to the case without an IAZ. Note that due to the large value of $M$ used, all active slip systems in the IAZ simulations are associated with slip transfer. Consequently, slip systems that are active in the IAZ simulations but not active in the simulations without an IAZ, would not have been expected based on a Schmid factor analysis.

To examine the effect of slip activity changes on lattice rotation, Fig. 5 compares the inverse pole figures (IPFs) at $60 \%$ reduction for the C-I interface with and without the IAZ. The initial orientation is shown in these IPFs for comparison. The results reveal that the lattice rotation in both crystals is much more pronounced with the IAZ, such that the C-I interface character is not maintained and is plastically unstable under rolling. Under the kinematic constraint of co-deformation alone (no IAZ), the C-I interface is metastable. The $\mathrm{Cu}$ crystal reorients $5-6^{\circ}$ to a final orientation that lies between the $\mathrm{C}$ and $\mathrm{D}$ texture components and the $\mathrm{Nb}$ phase essentially maintains its initial I orientation. Taken together, these results suggest that when slip continuity across an interface is enforced, the C-I interface is unstable with respect to rolling deformation. Thus, we conclude that so-called slip transfer is not predominant in the ARB material at submicron layer thicknesses.

The calculations are repeated for the D-I interface, an interface that closely neighbors the C-I interface in orientation space (by a disorientation of $7-8^{\circ}$ ). Like the C-I interface, it is frequently observed in the ARB material. Fig. 6 compares the $\mathrm{Cu}$ and Nb IPFs for the D-I interface with and without an IAZ. As shown, the D-I interface is also metastable when there is no IAZ, such that both crystals reorient slightly to attain a stable final orientation. With an IAZ, the D-I interface character is unstable. The $\mathrm{Cu}$ crystal reorients about the interface normal and the $\mathrm{Nb}$ crystal reorients significantly away from the I orientation. The slip activities for the D-I interface (and the subsequently studied interfaces as well) are given in the Appendix, where it is shown that, as before, the presence of an IAZ alters the slip activity as compared to simulations without an IAZ.

\subsection{Nano interfaces: Goss-I and Z-I}

The Goss-I and Z-I interface interfaces are observed in ARB composites after refinement to very fine layer thicknesses, $h=20 \mathrm{~nm}$ and less (Beyerlein et al., 2014a). These interface characters were present, 


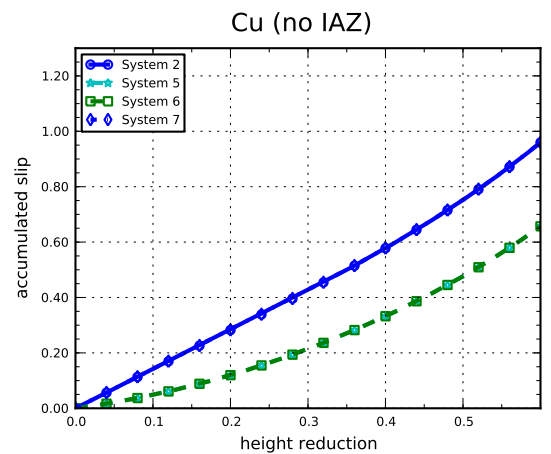

(a)

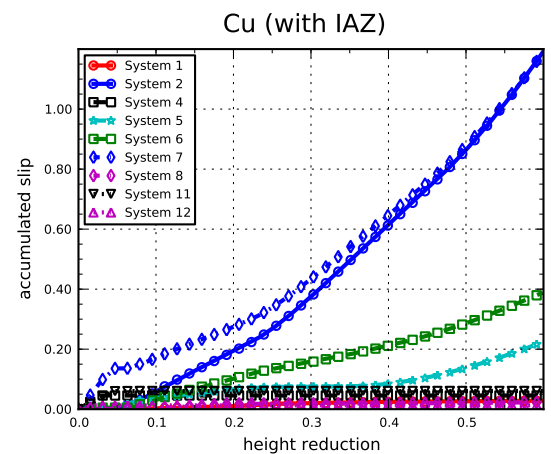

(c)

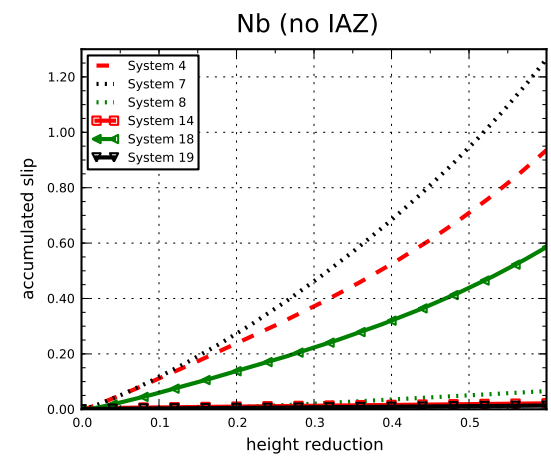

(b)

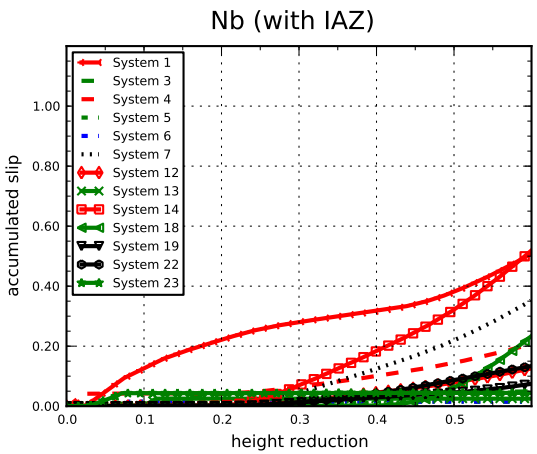

(d)

Figure 4: Accumulated slip histories for the C-I interface (a) Cu phase no IAZ (b) Nb phase no IAZ (c) $\mathrm{Cu}$ phase with IAZ and (d) Nb phase with IAZ. Note that the slip histories in (a) for systems $2 \& 7$ and $5 \& 6$, respectively, are identical.

although not as pronounced, in composites with submicron layer thicknesses. The calculations reveal that although the set of activated slip systems are different for simulations with and without the IAZ, the stable end states achieved by $60 \%$ reduction are nearly the same. The IPFs in Fig. 7 show that the Goss-I interface is perfectly stable as there is negligible lattice reorientation both with and without the IAZ. The IPFs in Fig. 8 reveal that the Z-I interface is unstable with the IAZ and metastable without the IAZ. More importantly, the Z-I interface reorients to become a near Goss-I interface in both cases, wherein the $\mathrm{Cu}$ crystal reorients to a near Goss orientation while the $\mathrm{Nb}$ crystal maintains a near I orientation. Experiments have shown that the C-I interfaces prevail in the submicron layered composites but not in the nanolayered composites. Our calculations reveal that with the IAZ, the Goss-I interface is stable and the C-I interface is not. This result implies that slip transfer may be more likely in the nanolayered materials thereby favoring the Goss-I interface.

\subsection{Secondary interface: Brass-I}

We examine the Brass-I interface as a last example. Although it is not a predominant interface in ARB composites, a non-negligible fraction of this interface exists in the ARB material at submicron layer thicknesses (Beyerlein et al., 2014a). The Brass-I interface is composed of two crystals that are well known to be stable in large strain rolling deformation, yet its frequency reduces as the layer thickness decreases into the nanolayer regime. Consistent with this observation, the Brass-I interface is shown to be perfectly stable under co-deformation alone (without an IAZ). Fig. 9 gives the IPFs for the $\mathrm{Cu}$ and $\mathrm{Nb}$ crystals with and without the IAZ. With an IAZ, the Brass-I interface is unstable. We find that the IAZ forces slip activity on systems that cause significant lattice reorientation. Even at modest levels of reduction, the $\mathrm{Cu}$ crystal has already reoriented approximately $30^{\circ}$. The $\mathrm{Nb}$ crystal has also already reoriented substantially at $15 \%$ reduction. This result supports the notion that the Brass-I interface 

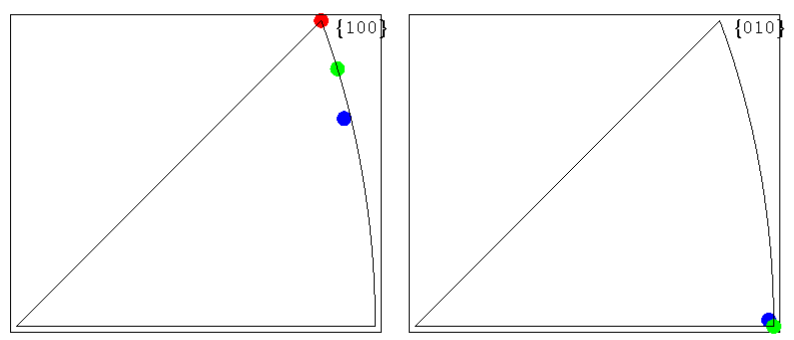

(a)
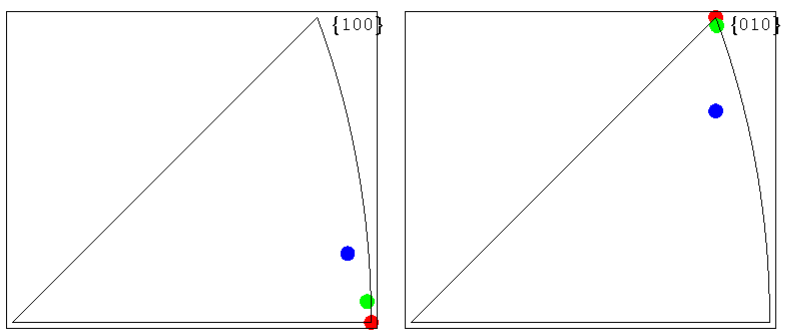

(b)
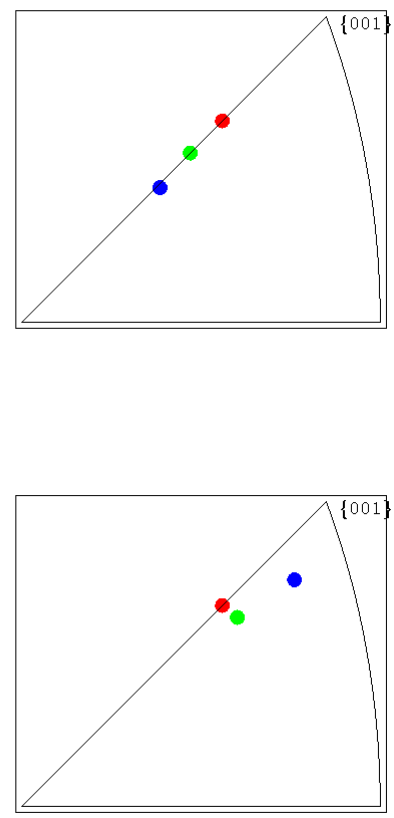

Figure 5: Inverse pole figures for the C-I interface (a) Cu phase (b) Nb phase after $60 \%$ reduction. The red points indicate the initial orientations, the green points indicate the results from the model without an IAZ, and the blue points indicate the results from the model with an IAZ.
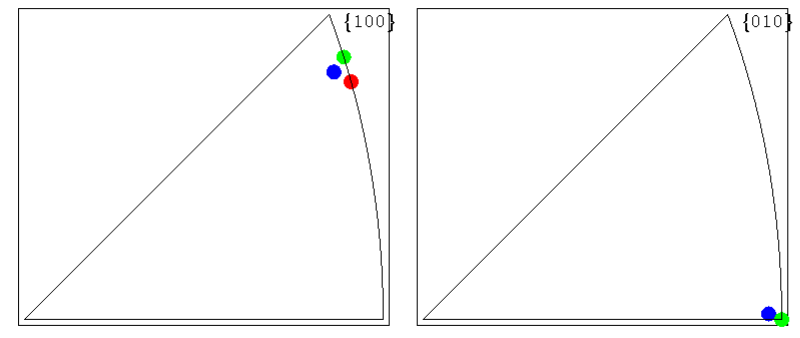

(a)
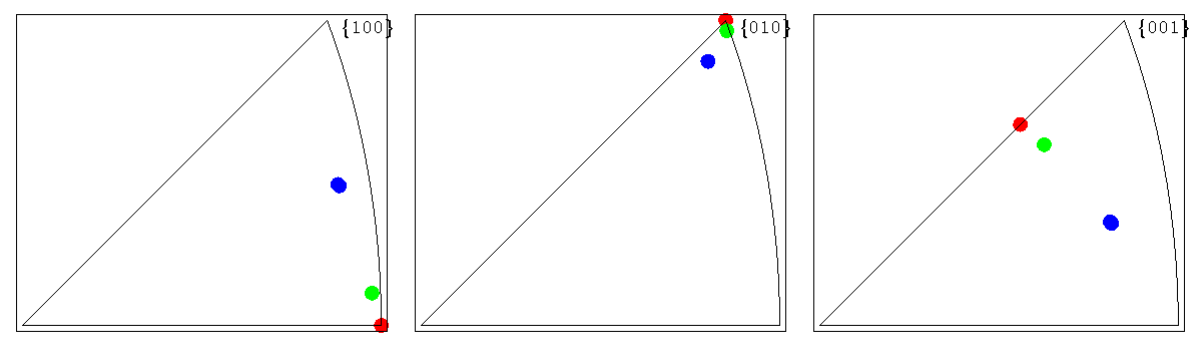

(b)

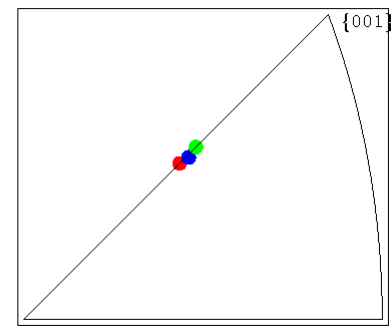
Figure 6: Inverse pole figures for the $\mathrm{D}-\mathrm{I}$ interface (a) Cu phase (b) Nb phase after $30 \%$ reduction. The
red points indicate the initial orientations, the green points indicate the results from the model without an IAZ, and the blue points indicate the results from the model with an IAZ. 

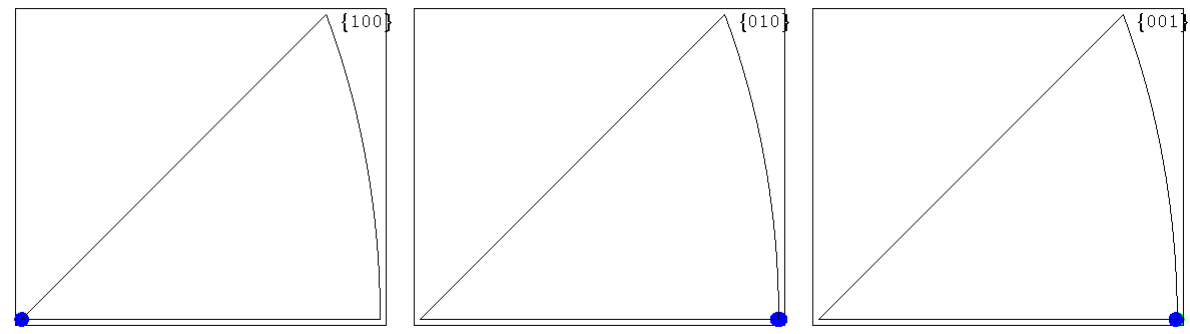

(a)
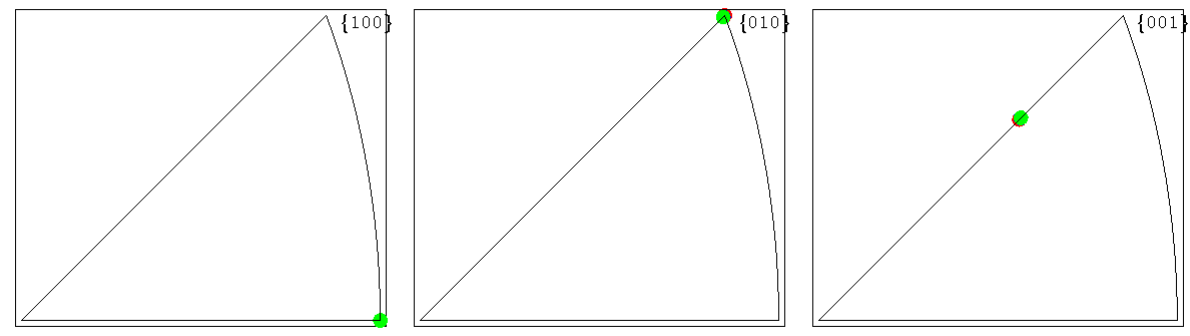

(b)

Figure 7: Inverse pole figures for the Goss-I interface (a) $\mathrm{Cu}$ phase (b) Nb phase after $60 \%$ reduction. The red points indicate the initial orientations, the green points indicate the results from the model without an IAZ, and the blue points indicate the results from the model with an IAZ. Note that all of the symbols are coincident due to negligible reorientation.
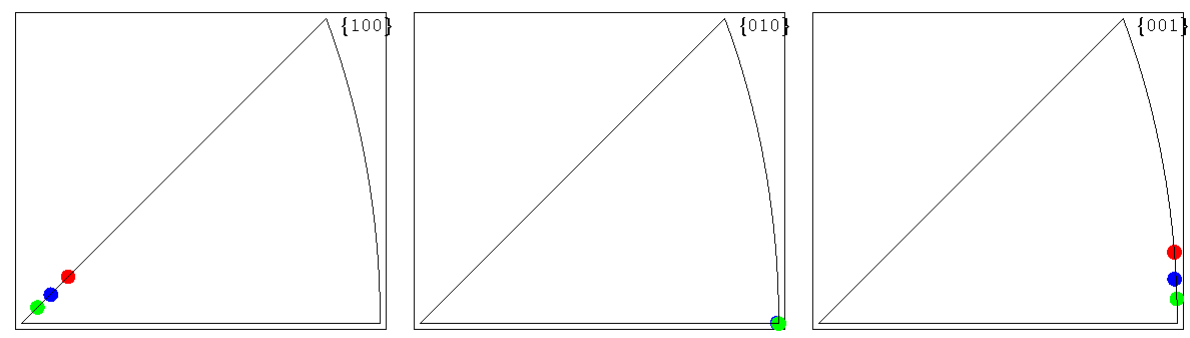

(a)
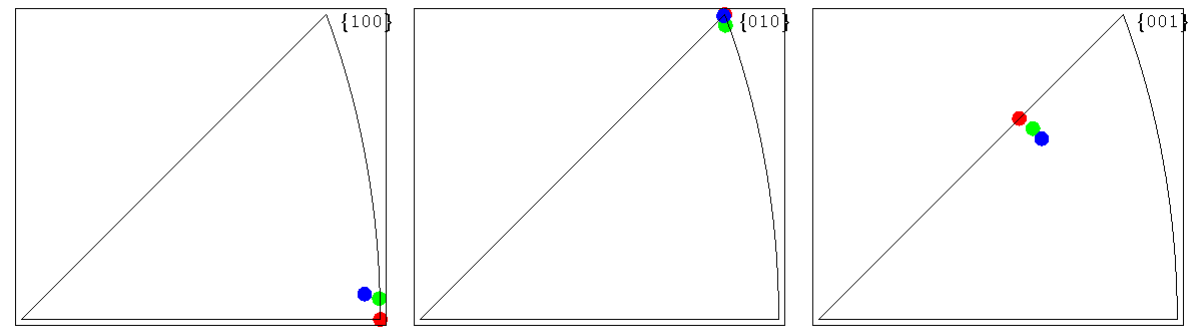

(b)

Figure 8: Inverse pole figures for the Z-I interface (a) Cu phase (b) Nb phase. The red points indicate the initial orientations, the green points indicate the results from the model without an IAZ, and the blue points indicate the results from the model with an IAZ. 

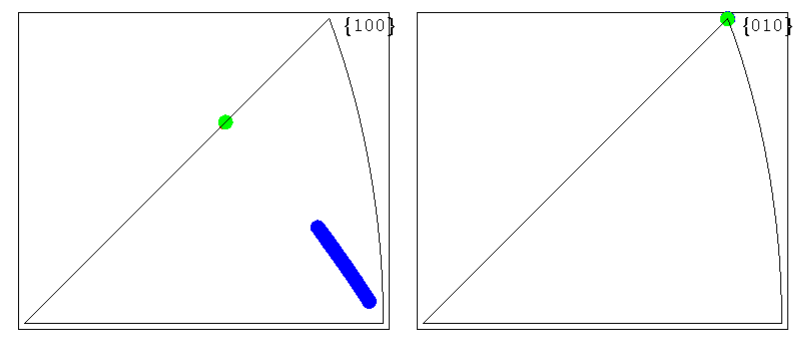

(a)
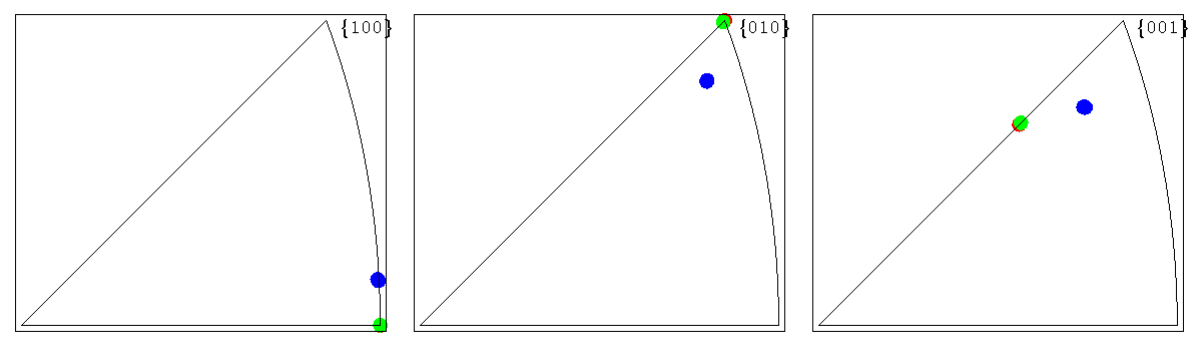

(b)
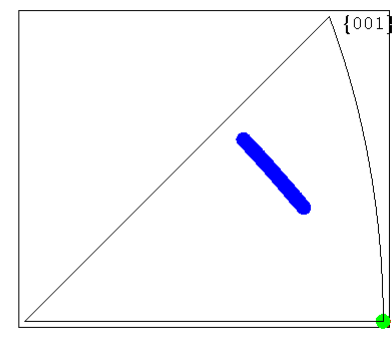

$$
\text { (a) }
$$

(a) $\mathrm{Cu}$ phase (b) Nb phase at $15 \%$ reduction. The Figure 9: Inverse pole figures for the Brass-I interface (a) Cuts
red points indicate the initial orientations, the green points indicate the results from the model without an IAZ, and the blue points indicate the results from the model with an IAZ. Note that the red and green symbols are coincident due to negligible reorientation for the case with no IAZ. Multiple blue points are shown for the $\mathrm{Cu}$ crystal of the Brass-I interface because it is deforming inhomogeneously.

would be expected to diminish in very fine scaled composites with $h$ in the nanoscale regime if slip continuity across the interface is enforced.

\section{Discussion}

To compare the plastic stability of the different interfaces, we calculate the amount of reorientation, $\Delta \theta$, for the crystals comprising the interface at a given strain. At each integration point, $\Delta \theta$ is determined from the elastic (lattice) rotation tensor, $\mathbf{R}^{e}$ using

$$
\Delta \theta=\arccos \left(\frac{\operatorname{tr} \mathbf{R}^{e}-1}{2}\right)
$$

where $\mathbf{R}^{e}$ is defined in the text following Eq. (3). The average of $\Delta \theta$ over each crystal yields a single value of $\Delta \theta$ for each point in the load history. We also consider a measure of the instantaneous plastic stability of the crystal, which depends on the time rate of change of $\Delta \theta$.

We introduce one plastic stability parameter denoted as $\Omega$ that is based on the average angle of lattice rotation in each crystal (Mayeur et al., 2013) for a given rolling strain, $\epsilon$, and is defined as

$$
\Omega=\exp \left(-\frac{\Delta \theta_{\mathrm{Cu}}}{\epsilon}\right) \exp \left(-\frac{\Delta \theta_{\mathrm{Nb}}}{\epsilon}\right)
$$

where $\Delta \theta_{\mathrm{Cu}}$ and $\Delta \theta_{\mathrm{Nb}}$ (Eq. 19) are the average angles of the lattice rotation within the $\mathrm{Cu}$ and $\mathrm{Nb}$ crystals, respectively, at a rolling strain of $\epsilon$. $\Omega$ varies from 0 to 1 , with 1 representing a perfectly stable interface (i.e., $\Delta \theta_{\mathrm{Cu}}=0$ and $\Delta \theta_{\mathrm{Nb}}=0$ ). Allowing for a small amount of crystal reorientation (the sum of the two crystal rotations is less than $10^{\circ}$ for the rolling strains considered in this work), implies that interfaces yielding $\Omega>0.8$ can be considered stable, those with $0.75<\Omega<0.8$ are metastable and those 
with $\Omega<0.75$ unstable. The magnitude of the true compressive strain, $\epsilon$ is given in terms of the rolling reduction, $R$, as

$$
\epsilon=|\ln (1-R)|
$$

The rate form of Eq. (20) is also a valuable measure of stability and can differentiate between crystals that have reached a stable configuration from those that will continue to reorient with further deformation. Therefore, we introduce an additional stability parameter, denoted as $\tilde{\Omega}$, as a measure of the instantaneous plastic stability of the interface. $\tilde{\Omega}$ is defined as

$$
\tilde{\Omega}=\exp \left(-\frac{\Delta \dot{\theta}_{\mathrm{Cu}}}{\dot{\epsilon}}\right) \exp \left(-\frac{\Delta \dot{\theta}_{\mathrm{Nb}}}{\dot{\epsilon}}\right)
$$

$\tilde{\Omega}$ also ranges between 0 and 1 , with 1 indicating a stable orientation at a given level of strain (null rotation rate). Non-zero rates represent an instability, although very slow rates of rotation (arbitrarily $>0.9$ ) could be associated with metastability.

Fig. 10 compares the interface stability parameters with (open symbols) and without (closed symbols) an IAZ for all of the studied cases. The different symbol shapes represent different values of rolling reduction, $R$ : $15 \%$ (circle), $30 \%$ (triangle), and 60\% (square). Recall, that $\Omega$ (Fig. 10(a)) quantifies the stability with respect to the initial interface orientations, whereas $\tilde{\Omega}$ (Fig. 10(b)) quantifies the stability with respect to the instantaneous crystal orientation. Considered together, the plastic stabilities



(a)

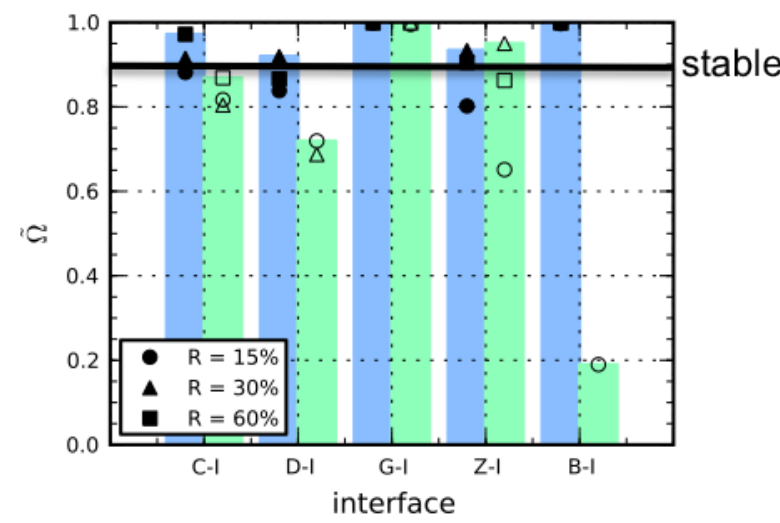

(b)

Figure 10: Interface stability parameters plotted for each of the considered interfaces (a) overall stability and (b) instantaneous stability at $60 \%$ reduction. Filled symbols (blue bars) are for simulations without an IAZ and open symbols (green bar) are for simulations with an IAZ.

as quantified by $\Omega$ and $\tilde{\Omega}$ suggest that the presence of an IAZ reduces the crystallographic stability of the C-I, D-I, and Brass-I interfaces. For these three interfaces, the different slip activity within the crystals induced by the IAZ forced the crystals to reorient seeking more stable orientations. In contrast, the changes in slip activity caused by the IAZ in the case of the Z-I and Goss-I interfaces did not significantly affect their stability. With or without an IAZ, the Goss-I interface remains stable and the Z-I interface reorients to the more stable Goss-I interface.

These findings suggest that C-I, D-I, and Brass-I interfaces are not stable when slip is biased towards systems that form favorable slip transfer pathways. In contrast, the plastic stability of the Goss-I and Z-I interfaces remain unaffected by such constraints and the Goss-I interface is found to be the most stable. This result provides a possible explanation for the change in interface character seen in ARB $\mathrm{Cu}-\mathrm{Nb}$ lamellar composites when layer thicknesses, $h$, refine below $50 \mathrm{~nm}$ (Zheng et al., 2013). When the layer thicknesses are submicron, the C-I and D-I interfaces are the primary interfaces and the Brass-I interface is secondary (Lee et al., 2012; Carpenter et al., 2012a,b). However, the Goss-I and Z-I interfaces become the dominant interfaces when $h$ reduces below $50 \mathrm{~nm}$ (Zheng et al., 2013). We surmise, based 
on the present results, that forced slip transfer across the interface at these very fine scales may have contributed to the prevalence of the Goss-I interface and loss of the C-I and D-I interfaces observed for the nanoscale composites with $h=10 \mathrm{~nm}$.

In the present calculations, we considered the IAZ to affect the entirety of a single crystalline layer. When the layers of the composite are polycrystalline, with many grains in the through thickness direction, the IAZ may extend only a finite distance into the layer, short of the full layer thickness. Only recently has some experimental work revealed the existence of an IAZ in these materials (Carpenter et al., 2014a) and that work suggests the IAZ would extend at least as much as one grain from the interface. Nothing in the current modeling paradigm prevents the prescription of a finite-sized IAZ. However, more experimental and theoretical work must be done to determine the size of the IAZ and its dependence on interface properties.

\section{Conclusions}

We developed a crystal plasticity modeling framework to capture the effects of slip transfer across material interfaces. The interface is assumed to restrict slip system availability by biasing the deformation to be accommodated by systems in the neighboring crystallites that are geometrically well-aligned and sufficiently stressed. The model is employed to study the plastic stability of $\mathrm{Cu}-\mathrm{Nb}$ heterophase interfaces under plane strain compression, an approximation of the deformation state during rolling, via crystal plasticity finite element simulations. The study focused on predominant interfaces that emerge in $\mathrm{ARB} \mathrm{Cu}-\mathrm{Nb}$ multilayer composites after extreme straining (the layer thickness below $1 \mu \mathrm{m}$ ) and revealed that deformation dominated by slip transfer reduces the plastic stability of most of the interfaces studied as compared to deformation dominated by interphase glide. Reduced plastic stability under dislocation transmission is the direct result of the higher constraints placed on slip system availability by the transmission criteria. Although the applications in this study focus on bimetal interfaces in layered composites, the model formulation is applicable to grain boundaries and bimetal interfaces in general polycrystalline materials.

\section{Acknowledgments}

JRM and IJB gratefully acknowledge support by the Center for Materials at Irradiation and Mechanical Extremes, an Energy Frontier Research Center funded by the US Department of Energy, Office of Science, Office of Basic Energy Sciences under Award Number 2008LANL1026. CAB and HMM acknowledge the support of the Los Alamos National Laboratory Directed Research and Development (LDRD) Project ER20140348. Los Alamos National Laboratory is operated by Los Alamos National Security LLC under DOE Contract DE AC52 06NA25396. 


\section{References}

Abaqus/Standard Version 6.9.1, 2007. Dassault systèmes simulia corp.

Acharya, A., 2007. Jump condition for gnd evolution as a constraint on slip transmission at grain boundaries. Philosophical Magazine 87 (8-9), 1349-1359.

Adams, B. L., Fullwood, D. T., Basinger, J., Hardin, T., 2012. High resolution ebsd-based dislocation microscopy. In: Materials Science Forum. Vol. 702. pp. 11-17.

Ardeljan, M., Beyerlein, I. J., Knezevic, M., 2014. A dislocation density based crystal plasticity finite element model: Application to a two-phase polycrystalline HCP/BCC composites. Journal of the Mechanics and Physics of Solids 66, 16-31.

Armstrong, R. W., Codd, I., Douthwaite, R. M., Petch, N. J., 1962. The plastic deformation of polycrystalline aggregates. Philosophical Magazine 7 (73), 45-58.

Ashmawi, W. M., Zikry, M. A., 2002. Prediction of grain-boundary interfacial mechanisms in polycrystalline materials. Journal of engineering materials and technology 124 (1), 88-96.

Beyerlein, I. J., Mara, N. A., Bhattacharyya, D., Necker, C. T., Alexander, D. J., 2011a. Texture evolution via combined slip and deformation twinning in rolled silver-copper eutectic nanocomposite. International Journal of Plasticity 27, 121-146.

Beyerlein, I. J., Mara, N. A., Carpenter, J. S., Nizolek, T., Mook, W. M., Wynn, T. A., McCabe, R. J., Mayeur, J. R., Kang, K., Zheng, S., et al., 2013a. Interface-driven microstructure development and ultra high strength of bulk nanostructured cu-nb multilayers fabricated by severe plastic deformation. Journal of Materials Research 28 (13), 1799-1812.

Beyerlein, I. J., Mayeur, J. R., McCabe, R. J., Zheng, S. J., Carpenter, J. S., Mara, N. A., $2014 a$. Influence of slip and twinning on the crystallographic stability of bimetal interfaces in nanocomposites under deformation. Acta Materialia 72, 137-147.

Beyerlein, I. J., Mayeur, J. R., Zheng, S., Mara, N. A., Wang, J., Misra, A., 2014b. Emergence of stable interfaces under extreme plastic deformation. Proceedings of the National Academy of Sciences, 201319436.

Beyerlein, I. J., McCabe, R. J., Tome, C. N., 2011b. Stochastic processes of $\{1012\}$ deformation twinning in hexagonal close-packed polycrystalline zirconium and magnesium. International Journal for Multiscale Computational Engineering 9 (4).

Beyerlein, I. J., Wang, J., Kang, K., Zheng, S. J., Mara, N. A., 2013b. Twinnability of bimetal interfaces in nanostructured composites. Materials Research Letters 1 (2), 89-95.

Beyerlein, I. J., Wang, J., Zhang, R., 2013c. Mapping dislocation nucleation behavior from bimetal interfaces. Acta Materialia 61 (19), 7488-7499.

Bieler, T. R., Eisenlohr, P., Roters, F., Kumar, D., Mason, D. E., Crimp, M., Raabe, D., 2009. The role of heterogeneous deformation on damage nucleation at grain boundaries in single phase metals. International Journal of Plasticity 25 (9), 1655-1683.

Bronkhorst, C. A., Hansen, B. L., Cerreta, E. K., Bingert, J. F., 2007. Modeling the microstructural evolution of metallic polycrystalline materials under localization conditions. Journal of the Mechanics and Physics of Solids 55 (11), 2351-2383.

Carpenter, J. S., Liu, X., Darbal, A., Nuhfer, N. T., McCabe, R. J., Vogel, S. C., LeDonne, J. E., Rollet, A. D., Barmak, K., Beyerlein, I. J., Mara, N. A., 2012a. A comparison of texture results obtained using precession electron diffraction and neutron diffraction methods at diminishing length scales in ordered bimetallic nanolamellar composites. Scripta Materialia 67, 336-339. 
Carpenter, J. S., McCabe, R. J., Mayeur, J. R., Mara, N. A., Beyerlein, I. J., 2014a. Interface-driven plasticity: The presence of an interface affected zone in metallic lamellar composites. Advanced Engineering Materials.

Carpenter, J. S., McCabe, R. J., Zheng, S. J., Wynn, T. A., Mara, N. A., Beyerlein, I. J., 2014 b. Processing parameter influence on texture and microstructural evolution in cu-nb multilayer composites fabricated via accumulative roll bonding. Metallurgical and Materials Transactions A, 1-17.

Carpenter, J. S., Vogel, S. C., LeDonne, J., Hammon, D. L., Beyerlein, I. J., Mara, N. A., 2012b. Bulk texture evolution of $\mathrm{Cu}-\mathrm{Nb}$ nanolamellar composites during accumulative roll bonding. Acta Materialia $60,1576-1586$.

Cermelli, P., Gurtin, M. E., 2002. Geometrically necessary dislocations in viscoplastic single crystals and bicrystals undergoing small deformations. International journal of solids and structures 39 (26), 6281-6309.

Chalmers, B., 1949. Some crystal-boundary phenomena in metals. Proceedings of the Royal Society of London. Series A. Mathematical and Physical Sciences 196 (1044), 64-73.

Chookajorn, T., Murdoch, H. A., Schuh, C. A., 2012. Design of stable nanocrystalline alloys. Science 337 (6097), 951-954.

Clark, W. A. T., Wagoner, R. H., Shen, Z. Y., Lee, T. C., Roberston, I. M., Birnbaum, H. K., 1992. On the criteria for slip transmission across interfaces in polycrystals. Scripta Metallurgica et Materialia 26 (2), 203-206.

Davis, K. G., Teghtsoonian, E., Lu, A., 1966. Slip band continuity across grain boundaries in aluminum. Acta Metallurgica 14 (12), 1677-1684.

Ekh, M., Bargmann, S., Grymer, M., 2011. Influence of grain boundary conditions on modeling of sizedependence in polycrystals. Acta mechanica 218 (1-2), 103-113.

Embury, J. D., Hirth, J. P., 1994. On dislocation storage and the mechanical response of fine scale microstructures. Acta metallurgica et materialia 42 (6), 2051-2056.

Evers, L. P., Brekelmans, W. A. M., Geers, M. G. D., 2004. Scale dependent crystal plasticity framework with dislocation density and grain boundary effects. International Journal of solids and structures 41 (18), 5209-5230.

Gleiter, H., 2008. Our thoughts are ours, their ends none of our own: Are there ways to synthesize materials beyond the limitations of today? Acta Materialia 56 (19), 5875-5893.

Hammelrath, H., Butler, Jr., J. F., Juul-Jensen, D., Leffers, T., Hu, H., Lücke, K., 1991. An odf study of the deformation and recrystallization textures of rolled and channel-die compressed high purity copper. Textures and Microstructures 13, 165-187.

Hansen, B., Carpenter, J., Sintay, S., Bronkhorst, C., McCabe, R., Mayeur, J., Mourad, H., Beyerlein, I., Mara, N., Chen, S., III, G. G., 2013. Modeling the texture evolution of $\mathrm{Cu} / \mathrm{Nb}$ layered composites during rolling. International Journal of Plasticity 49 (0), $71-84$.

Hirth, J. P., 1972. The influence of grain boundaries on mechanical properties. Metallurgical Transactions 3 (12), 3047-3067.

Hook, R. E., Hirth, J. P., 1967a. The deformation behavior of isoaxial bicrystals of Fe-3\% si. Acta Metallurgica 15 (3), 535-551.

Hook, R. E., Hirth, J. P., 1967b. The deformation behavior of non-isoaxial bicrystals of Fe-3\% si. Acta Metallurgica 15 (7), 1099-1110.

Huang, H., Spaepen, F., 2000. Tensile testing of free-standing $\mathrm{Cu}, \mathrm{Ag}$ and $\mathrm{Al}$ thin films and $\mathrm{Ag} / \mathrm{Cu}$ multilayers. Acta Materialia 48 (12), 3261-3269. 
Hunter, A., Beyerlein, I. J., 2013. Unprecedented grain size effect on stacking fault width. APL Materials $1(3), 032109$.

Keh, A. S., Weissmann, S., 1963. Deformation substructure in body-centered cubic metals. Electron Microscopy and Strength of Crystals, 231-300.

Kocks, U. F., Mecking, H., 2003. Physics and phenomenology of strain hardening: the FCC case. Progress in Materials Science 48 (3), 171-273.

Lebensohn, R. A., 2001. N-site modeling of a 3d viscoplastic polycrystal using fast fourier transform. Acta Materialia 49 (14), 2723-2737.

Lee, S.-B., LeDonne, J., Lim, S., Beyerlein, I., Rollett, A., 2012. The heterophase interface character distribution of physical vapor-deposited and accumulative roll-bonded cu-nb multilayer composites. Acta Materialia 60 (4), $1747-1761$.

Lee, T. C., Robertson, I. M., Birnbaum, H. K., 1990a. An in situ transmission electron microscope deformation study of the slip transfer mechanisms in metals. Metallurgical Transactions A 21 (9), $2437-2447$.

Lee, T. C., Robertson, I. M., Birnbaum, H. K., 1990b. Tem in situ deformation study of the interaction of lattice dislocations with grain boundaries in metals. Philosophical Magazine A 62 (1), 131-153.

Li, J. C. M., 1963. Petch relation and grain boundary sources. Transactions of the Metallurgical Society of AIME 227 (1), 239-247.

Lim, H., Lee, M., Kim, J., Adams, B., Wagoner, R., 2011. Simulation of polycrystal deformation with grain and grain boundary effects. International Journal of Plasticity 27 (9), 1328-1354.

Louat, N., 1985. Alloys, strong at room and elevated temperatures from powder metallurgy. Acta Metallurgica 33 (1), 59-69.

Lu, L., Chen, X., Huang, X., Lu, K., 2009. Revealing the maximum strength in nanotwinned copper. Science 323 (5914), 607-610.

Ma, A., Roters, F., Raabe, D., 2006. On the consideration of interactions between dislocations and grain boundaries in crystal plasticity finite element modeling-theory, experiments, and simulations. Acta materialia 54 (8), 2181-2194.

Mara, N. A., Bhattacharyya, D., Dickerson, P., Hoagland, R. G., Misra, A., 2008. Deformability of ultrahigh strength $5 \mathrm{~nm} \mathrm{Cu} / \mathrm{Nb}$ nanolayered composites. Applied Physics Letters 92 (23), 231901231903.

Mayeur, J. R., Beyerlein, I. J., Bronkhorst, C. A., Mourad, H. M., 2014. The influence of grain interactions on the plastic stability of heterophase interfaces. Materials 7 (1), 302-322.

Mayeur, J. R., Beyerlein, I. J., Bronkhorst, C. A., Mourad, H. M., Hansen, B. L., 2013. A crystal plasticity study of heterophase interface character stability of $\mathrm{cu} / \mathrm{nb}$ bicrystals. International Journal of Plasticity 48 (0), $72-91$.

Meissonnier, F. T., Busso, E. P., O’Dowd, N. P., 2001. Finite element implementation of a generalised non-local rate-dependent crystallographic formulation for finite strains. International journal of plasticity 17 (4), 601-640.

Mishin, Y., Asta, M., Li, J., 2010. Atomistic modeling of interfaces and their impact on microstructure and properties. Acta Materialia 58 (4), 1117-1151.

Misra, A., 2006. Nanostructure Control of Materials. Taylor \& Francis.

Misra, A., Hirth, J. P., Hoagland, R. G., 2005. Length-scale-dependent deformation mechanisms in incoherent metallic multilayered composites. Acta Materialia 53 (18), 4817-4824. 
Monclús, M. A., Zheng, S. J., Mayeur, J. R., Beyerlein, I. J., Mara, N. A., Polcar, T., Llorca, J., MolinaAldareguía, J. M., 2013. Optimum high temperature strength of two-dimensional nanocomposites. APL Materials 1 (5), 052103.

Niezgoda, S. R., Kanjarla, A. K., Beyerlein, I. J., Tomé, C. N., 2014. Stochastic modeling of twin nucleation in polycrystals: An application in hexagonal close-packed metals. International Journal of Plasticity 56, 119-138.

Patriarca, L., Abuzaid, W., Sehitoglu, H., Maier, H. J., 2013. Slip transmission in bcc fecr polycrystal. Materials Science and Engineering: A 588, 308-317.

Robertson, I. M., Lee, T. C., Rozenak, P., Bond, G. M., Birnbaum, H. K., 1989. Dynamic observations of the transfer of slip across a grain boundary. Ultramicroscopy 30 (1), 70-75.

Roters, F., Eisenlohr, P., Hantcherli, L., Tjahjanto, D. D., Bieler, T. R., Raabe, D., 2010. Overview of constitutive laws, kinematics, homogenization and multiscale methods in crystal plasticity finiteelement modeling: Theory, experiments, applications. Acta Materialia 58 (4), 1152-1211.

Schweitz, K. O., Chevallier, J., Bottiger, J., Matz, W., Schell, N., 2001. Hardness in Ag/Ni, Au/Ni and $\mathrm{Cu} / \mathrm{Ni}$ multilayers. Philosophical Magazine A 81 (8), 2021-2032.

Seal, J. R., Crimp, M. A., Bieler, T. R., Boehlert, C. J., 2012. Analysis of slip transfer and deformation behavior across the $\alpha / \beta$ interface in ti-5al-2.5 sn (wt.\%) with an equiaxed microstructure. Materials Science and Engineering: A 552, 61-68.

Šittner, P., Novak, V., Bradler, J., 1992. Persistent slip band-grain boundary interactions in low strain fatigue of isoaxial fe-14wt.\% cr bicrystals. Scripta metallurgica et materialia 27 (6), 705-710.

Šittner, P., Paidar, V., 1989. Observation and interpretation of grain boundary compatibility effects in fe-3.3 wt\% si bicrystals. Acta Metallurgica 37 (7), 1717-1726.

Spearot, D. E., Tschopp, M. A., Jacob, K. I., McDowell, D. L., 2007. Tensile strength of $\langle 100\rangle$ and $\langle 110\rangle$ tilt bicrystal copper interfaces. Acta Materialia 55 (2), 705-714.

van der Sluis, O., Schreurs, P. J. G., Brekelmans, W. A. M., Meijer, H. E. H., 2000. Overall behaviour of heterogeneous elastoviscoplastic materials: effect of microstructural modelling. Mechanics of Materials $32(8), 449-462$.

Voyiadjis, G. Z., Faghihi, D., Zhang, Y., 2014. A theory for grain boundaries with strain-gradient plasticity. International Journal of Solids and Structures 51 (10), 1872-1889.

Wang, J., Hoagland, R. G., Hirth, J. P., Misra, A., 2008. Atomistic modeling of the interaction of glide dislocations with "weak" interfaces. Acta Materialia 56 (19), 5685-5693.

Wang, L., Eisenlohr, P., Yang, Y., Bieler, T. R., Crimp, M. A., 2010a. Nucleation of paired twins at grain boundaries in titanium. Scripta Materialia 63 (8), 827-830.

Wang, L., Yang, Y., Eisenlohr, P., Bieler, T. R., Crimp, M. A., Mason, D. E., 2010b. Twin nucleation by slip transfer across grain boundaries in commercial purity titanium. Metallurgical and Materials Transactions A 41 (2), 421-430.

Werner, E., Prantl, W., 1990. Slip transfer across grain and phase boundaries. Acta Metallurgica et Materialia 38 (3), 533-537.

Werner, E., Stüwe, H. P., 1985a. On the work-hardening of dual-phase alloys. Zeitschrift fur Metallkunde $76(5), 353-357$.

Werner, E., Stüwe, H. P., 1985b. Phase boundaries as obstacle to dislocations motion. Materials Science and Engineering 68 (2), 175-182.

Zhang, R. F., Wang, J., Beyerlein, I. J., Misra, A., Germann, T. C., 2012. Atomic-scale study of nucleation of dislocations from fcc-bcc interfaces. Acta Materialia 60, 2855-2865. 
Zheng, S., Beyerlein, I. J., Carpenter, J. S., Kang, K., Wang, J., Han, W., Mara, N. A., 2013. Highstrength and thermally stable bulk nanolayered composites due to twin-induced interfaces. Vol. 4.

Zheng, S. J., Beyerlein, I. J., Wang, J., Carpenter, J. S., Han, W. Z., Mara, N. A., 2012. Deformation twinning mechanisms from bi-metal interfaces as revealed by in-situ straining in the TEM. Acta Materialia 60, 5858-5866.

Zhu, Y. T., Liao, X. Z., Wu, X. L., 2012. Deformation twinning in nanocrystalline materials. Progress in Materials Science 57 (1), 1-62. 
Appendix A. Slip system nomenclature

Table A.4: Slip system nomenclature (fcc).

\begin{tabular}{ccc}
\hline \hline System & Plane Normal & Slip Direction \\
\hline 1 & $(111)$ & {$[1 \overline{1} 0]$} \\
2 & $(111)$ & {$[10 \overline{1}]$} \\
3 & $(111)$ & {$[01 \overline{1}]$} \\
4 & $(\overline{1} 11)$ & {$[101]$} \\
5 & $(\overline{1} 11)$ & {$[110]$} \\
6 & $(\overline{1} 11)$ & {$[01 \overline{1}]$} \\
7 & $(1 \overline{1} 1)$ & {$[10 \overline{1}]$} \\
8 & $(1 \overline{1} 1)$ & {$[011]$} \\
9 & $(1 \overline{1} 1)$ & {$[110]$} \\
10 & $(11 \overline{1})$ & {$[1 \overline{1} 0]$} \\
11 & $(11 \overline{1})$ & {$[101]$} \\
12 & $(11 \overline{1})$ & {$[011]$} \\
\hline \hline
\end{tabular}

Table A.5: Slip system nomenclature (bcc).

\begin{tabular}{cccccc}
\hline \hline System & Plane Normal & Slip Direction & System & Plane Normal & Slip Direction \\
\hline 1 & $(110)$ & {$[1 \overline{1} 1]$} & 13 & $(112)$ & {$[11 \overline{1}]$} \\
2 & $(110)$ & {$[\overline{1} 11]$} & 14 & $(\overline{1} 12)$ & {$[1 \overline{1} 1]$} \\
3 & $(011)$ & {$[11 \overline{1}]$} & 15 & $(1 \overline{1} 2)$ & {$[\overline{1} 11]$} \\
4 & $(011)$ & {$[1 \overline{1} 1]$} & 16 & $(11 \overline{2})$ & {$[1 \overline{1} 1]$} \\
5 & $(101)$ & {$[1 \overline{1} \overline{1}]$} & 17 & $(121)$ & {$[1 \overline{1} 0]$} \\
6 & $(101)$ & {$[\overline{1} 11]$} & 18 & $(\overline{1} 21)$ & {$[11 \overline{1}]$} \\
7 & $(\overline{1} 10)$ & {$[111]$} & 19 & $(1 \overline{2} 1)$ & {$[111]$} \\
8 & $(\overline{1} 10)$ & {$[1 \overline{1} \overline{1}]$} & 20 & $(12 \overline{1})$ & {$[\overline{1} 11]$} \\
9 & $(0 \overline{1} 1)$ & {$[111]$} & 21 & $(211)$ & {$[\overline{1} 11]$} \\
10 & $(0 \overline{1} 1)$ & {$[\overline{1} 11]$} & 22 & $(\overline{2} 11)$ & {$[111]$} \\
11 & $(\overline{\overline{1}} 01)$ & {$[11 \overline{1}]$} & 23 & $(2 \overline{1} 1)$ & {$[11 \overline{1}]$} \\
12 & $(\overline{1} 01)$ & {$[1 \overline{1} 1]$} & 24 & $(21 \overline{1})$ & {$[1 \overline{1} 1]$} \\
\hline \hline
\end{tabular}




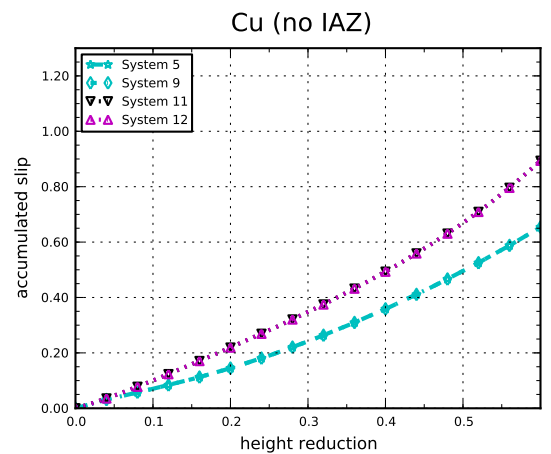

(a)

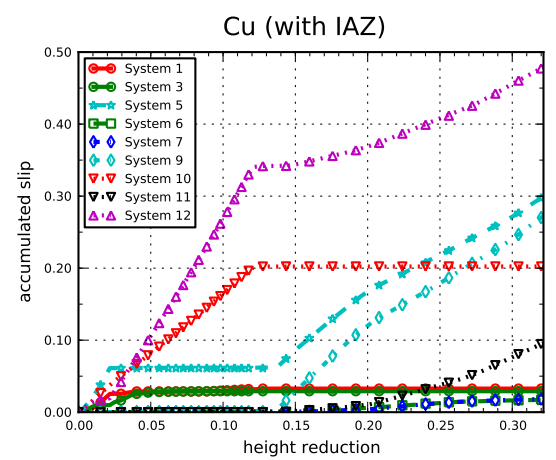

(c)

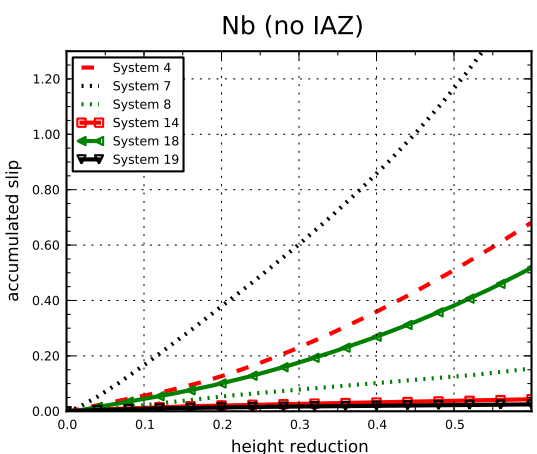

(b)

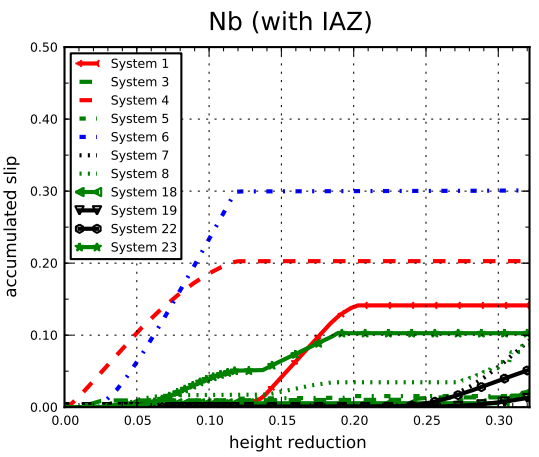

(d)

Figure B.11: Accumulated slip histories for the D-I interface (a) Cu phase no IAZ (b) Nb phase no IAZ (c) $\mathrm{Cu}$ phase with IAZ and (d) Nb phase with IAZ. 


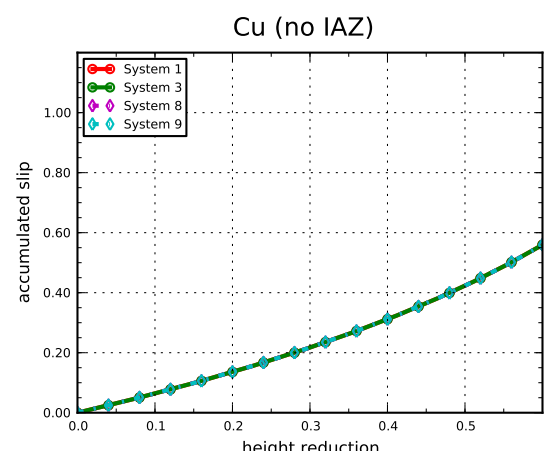

(a)

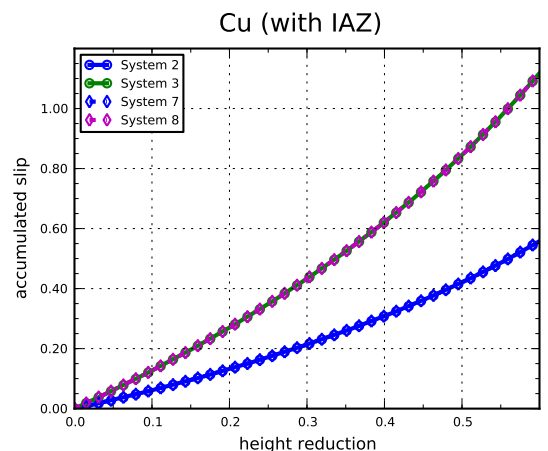

(c)

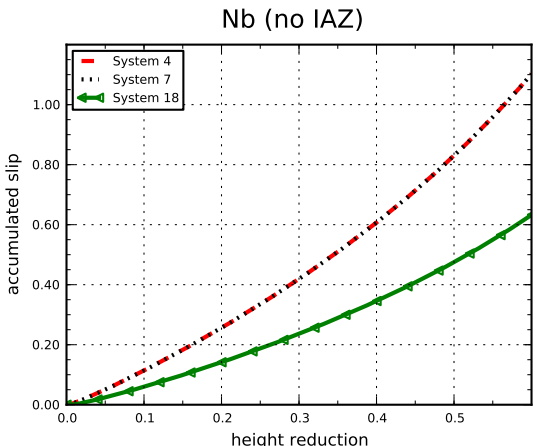

(b)

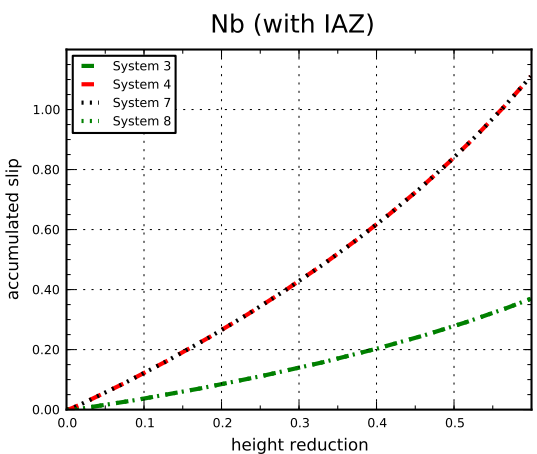

(d)

Figure B.12: Accumulated slip histories for the Goss-I interface (a) Cu phase no IAZ (b) Nb phase no IAZ (c) $\mathrm{Cu}$ phase with IAZ and (d) Nb phase with IAZ. 


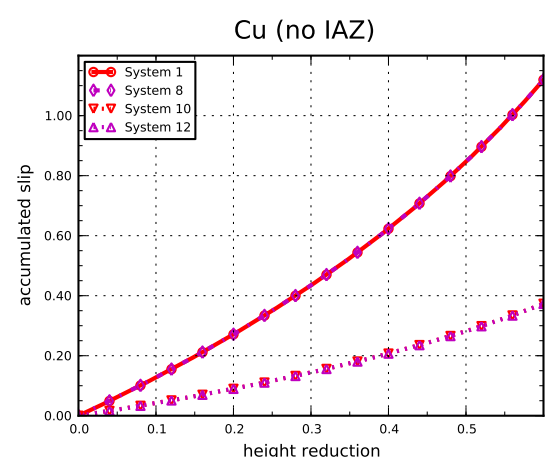

(a)

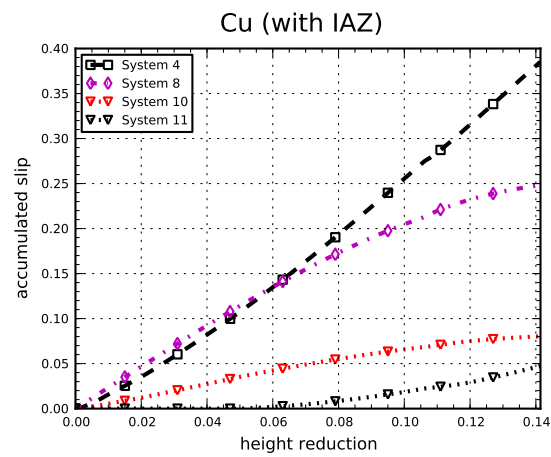

(c)

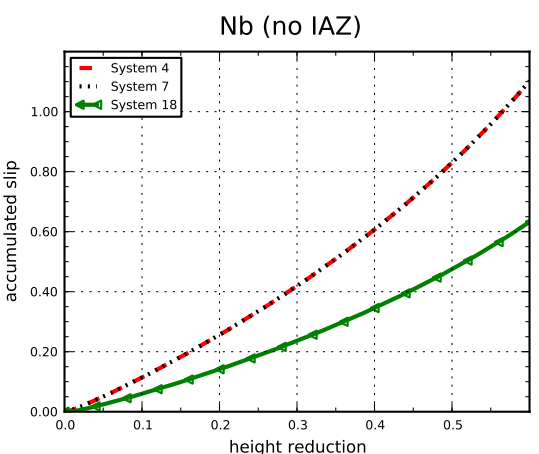

(b)

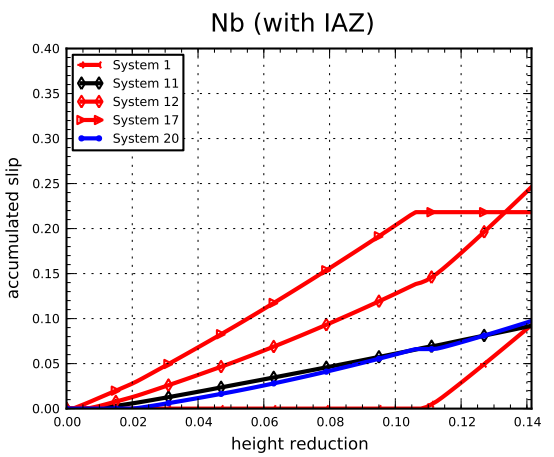

(d)

Figure B.13: Accumulated slip histories for the Brass-I interface (a) Cu phase no IAZ (b) Nb phase no IAZ (c) $\mathrm{Cu}$ phase with IAZ and (d) Nb phase with IAZ. 


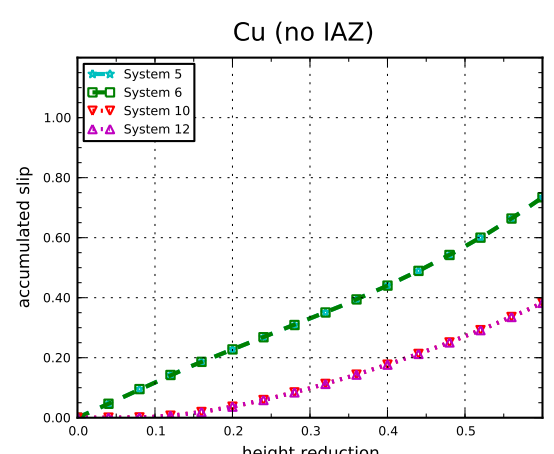

(a)

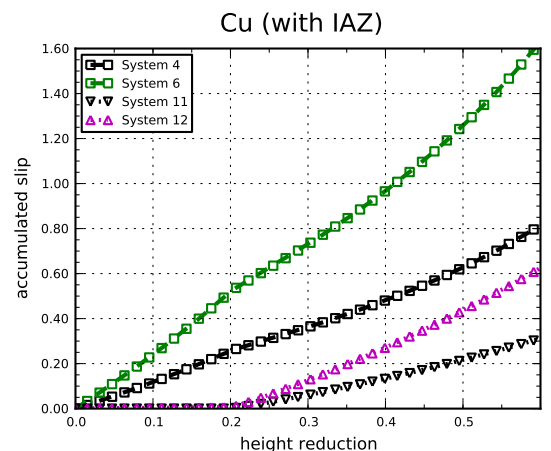

(c)

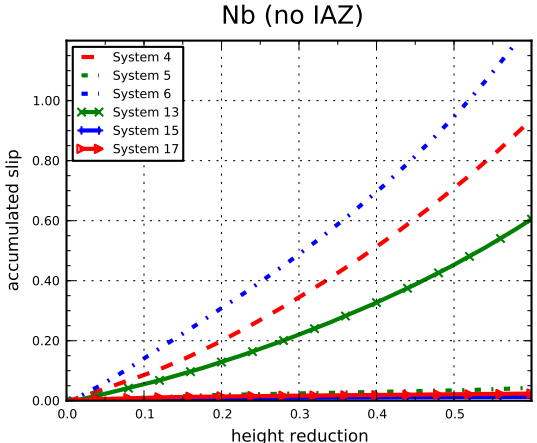

(b)

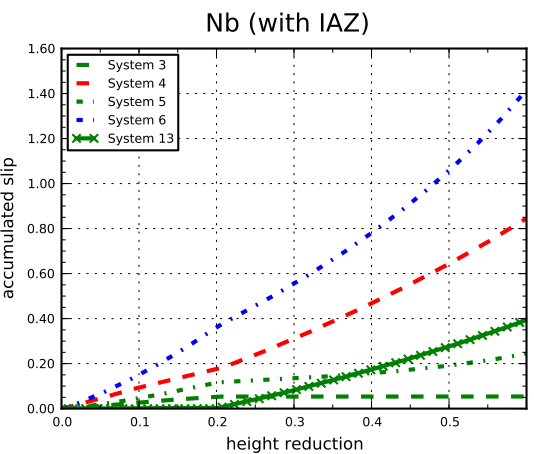

(d)

Figure B.14: Accumulated slip histories for the Z-I interface (a) Cu phase no IAZ (b) Nb phase no IAZ (c) $\mathrm{Cu}$ phase with IAZ and (d) Nb phase with IAZ. 